Pacific Journal of Mathematics

INTERMEDIATE RIESZ SPACES 


\section{INTERMEDIATE RIESZ SPACES}

\section{J. QUINN}

A Riesz space $E$ will be defined to be intermediate with respect to the Riesz space $L$ if $L$ can be embedded in $E$ as a Riesz subspace $L^{*}$ in such a way that the elements of $E$ are both the infimum and the supremum of elements in $L^{*}$. The main objectives of this paper are to investigate the extendability of certain order convergence properties from a Riesz space to its intermediate spaces and to compare the prime ideal structure of a Riesz space to the prime ideal structure of its intermediate spaces.

With regards to the first objective, one of the most useful intermediate Riesz spaces investigated is the order cauchy completion. The sequence $\left\{x_{n}\right\}$ in $L$ is order cauchy provided there exists $y_{n} \downarrow 0$ in $L$ such that, for $m \geqq n,\left|x_{n}-x_{m}\right| \leqq y_{n}$. L is order cauchy complete provided every order cauchy sequence converges. The order cauchy completion of $L$ is a Riesz space $L_{o c}^{-}$which is intermediate with respect to $L$ and each of whose elements is both the supremum of a countable subset of $L$ and the infimum of a countable subset of $L$. It is shown that such properties as the Egoroff property, the diagonalization property, and regularity are possessed by $L$ if and only if possessed by $L_{o c}^{-}$. This result is shown to be, in some sense, the best possible result. With regards to the second objective, if $E$ is intermediate with respect to $L$ and if $I$ is an ideal in $L$ then by $I(E)$ is denoted the ideal in $E$ generated by $I$. $L$ has the prime extension property with respect to $E$ if $P(E)$ is prime in $E$ whenever $P$ is a prime in $L$. One of the central results implies that $L$ has the prime extension property with respect to $E$ if and only if whenever $e_{1}, e_{2} \in E^{+}$are such that $e_{1} \wedge e_{2}=0$ there exist $y_{1}, y_{2} \in L$ with $y_{1} \geqq e_{1}$ and $y_{2} \geqq e_{2}$ such that $y_{1} \wedge y_{2}=0$.

If an Archimedean Riesz space $L$ is order separable, then it is known that its Dedekind completion $L^{\wedge}$ is order separable as well. Even more, order convergence in $L$ and $L^{\wedge}$ are (under these circumstances) very strongly related in the sense that if $\left\{x_{n}\right\} \subset L^{\wedge}$ is a sequence in $L^{\wedge}$ such that $x_{n} \downarrow 0$ then there exists a sequence $\left\{y_{n}\right\} \subset L$ with $y_{n} \downarrow 0$ such that, for each $n, x_{n} \leqq y_{n}$. These two facts were used in [4] to show that the diagonal property extends from an order separable Archimedean Riesz space to its Dedekind completion. These same facts were used in [16] to obtain analagous results for several other order convergence properties. Some studies along a different vein in [13] and [8] involved comparing the prime ideal structure or the band 
structure of an Archimedean Riesz space with the corresponding structure in its Dedekind completion. The property that the Dedekind completion $L^{\wedge}$ of an Archimedean Riesz space $L$ has which made the above comparisons possible is the property that $L$ can be embedded in $L^{\wedge}$ in such a way that each of $L^{\wedge}$ 's elements is both the infimum and supremum of elements in $L$ 's embedded copy. It seems reasonable to suppose, therefore, that comparisons as were made in [8] and [13] can be made for any two Riesz spaces which are related in this way. With respect to the problem of obtaining a coherent theory for the extendability of order convergence properties from a Riesz space to one containing it, one would at least want to assume that the two Riesz spaces are related in the manner mentioned above. However, it should also be clear that the Dedekind completion of an Archimedean Riesz space is in general not very appropriate for this kind of a study especially when the order convergence properties being considered are sequential in nature.

In the sequel, the Riesz space $E$ will be defined to be intermediate with respect to the Riesz space $L$ if $L$ can be embedded in $E$ as a Riesz subspace $L^{*}$ in such a way that the elements of $E$ are the infimum and the supremum of elements in $L^{*}$. As already mentioned, our main objectives are to investigate the extendability of certain order convergence properties from a Riesz space to its intermediate spaces and to compare the prime ideal structure of a Riesz space to the prime ideal structure of its intermediate spaces. The main results pertaining to the former objective are to be found in $\$ 6$. The main results pertaining to the latter objective are in $\S 10$. In $\S 1$, we define the concept of an intermediate Riesz space and discuss the general theory of such spaces. The concept of the intermediate completion of a Riesz space is also introduced here. The discussion concerning the intermediate completion is very brief since the basic theory can be extracted from several papers on lattice ordered groups. Section 2 is used to introduce three intermediate Riesz spaces of particular interest, one of which (the order cauchy completion) is the intermediate Riesz space most appropriate for investigating the extendabiltiy of sequential order convergence properties.

The approach used to establish the existence and uniqueness of these spaces is a very general one and does not give much insight into their structure. To a large extent this could be remedied by appeal to the literature. However, not every property we need to establish can be so obtained (at least not conveniently). The theory of upper and lower elements contained in $\$ \S 3$ and 4 , which is reminiscent of that theory to be found in $\$ 60$ of $\left[9\right.$, Note $\left.\mathrm{XVI}_{\mathrm{A}}\right]$, not only provides all the structure results obtainable from the literature but in addition provides several other results which are both interesting in themselves and are needed to obtain some of our main results in $\S \S 6$ and 10 . 
Sections 5 and 7 are totally given over to examples. Section 8 gives sufficient conditions in terms of certain projection properties for the order cauchy completion of an Archimedean Riesz space to be Dedekind complete or Dedekind- $\sigma$-complete. One of these results is particularly important for one of the main results in $\S 10$. In $\S 9$ we give two interesting applications to the theory of topological vector lattices of some of the concepts considered previous to this section.

1. Intermediate Riesz spaces. In this section we will define and discuss briefly the general concept of an intermediate Riesz space. In conjunction with this discussion we will discuss the concept and theory of the intermediate completion of an arbitrary Riesz space.

Definition 1.1. Let $L$ be a Riesz space. The Riesz space $E$ is said to be intermediate with respect to $L$ if there exists a Riesz isomorphism $\phi$ embedding $L$ as a Riesz subspace of $E$ such that, for each $e \in E$,

$$
e=\sup \{\phi(x): x \in L, \phi(x) \leqq e\}=\inf \{\phi(x): x \in L, \phi(x) \geqq e\} .
$$

The Riesz isomorphism $\phi$ will be referred to as an intermediate embedding.

The MacNeille conditional completion of a partially ordered set as applied to a vector lattice $L$ (we denote $L^{\prime}$ 's completion by $L^{\wedge}$ ) consists of the cuts $[A, B]$ in $L$ such that $A \neq \phi$ and $B \neq \phi$ (see [12]). $L$ can be very naturally embedded in $L^{\wedge}$ and, assuming this has been done, each element $\hat{x}$ in $L^{\wedge}$ has the form $\hat{x}=\sup \{x \in L: x \leqq \hat{x}\}=$ $\inf \{y \in L: y \geqq \hat{x}\}$. In general, $L^{\wedge}$ fails to be a vector lattice (indeed, it is a vector lattice if and only if $L$ is Archimedean). Everett [5] introduced (for lattice ordered groups) a related space which tends to correct this shortcoming. For a more complete discussion of Everett's space than that contained in [5], the reader is referred to [3]. To obtain Everett's extension we begin with $L^{\wedge}$. It was proved in [5, Th. 6, p. 115] that the element $\hat{x}$ in $L^{\wedge}$ has an additive inverse if and only if $\inf \{b-a: b, a \in L$ with $b \geqq \hat{x}$ and $a \leqq \hat{x}\}=0$. The Everett extension $L^{*}$ of $L$ then is taken to be the set of all elements of $L^{\wedge}$ which have an additive inverse. It is not difficult to see that $L^{*}$ is a vector lattice which is intermediate with respect to $L$.

We make a definition.

Definition 1.2. Let $L$ be a Riesz space. The Riesz space $K$ is said to be an intermediate completion of $L$ if the following conditions are satisfied:

(i) $K$ is intermediate with respect to $L$.

(ii) If $A$ is any bounded above subset of $K$ and $U$ is the set of all 
upper bounds for $A$ then $\sup A$ exists in $K$ if and only if inf $\{u-a$ : $u \in U$ and $a \in A\}=0$.

We have already seen that intermediate completions exist, since the Everett extension is such a space.

In fact, using standard techniques, the following proposition can be established.

Proposition 1.3. Let $L$ be a Riesz space and let $L_{1}$ and $L_{2}$ be intermediate completions of L. Furthermore, let $\phi_{1}$ and $\phi_{2}$ be intermediate embeddings of $L$ into $L_{1}$ and $L_{2}$, respectively. Then, there exists a Riesz isomorphism $\psi$ of $L_{1}$ onto $L_{2}$ such that $\psi / \phi_{1}(L)=\phi_{2}^{\circ} \phi_{1}^{-1}$.

COROllary 1.4. Every Riesz space has a unique up to lattice isomorphism intermediate completion.

In the sequel, we will denote the intermediate completion of a Riesz space $L$ by $L^{*}$. We will denote the Dedekind completion of an Archimedean Riesz space $L$ by $L^{\wedge}$. The reader should keep in mind that if $L$ is Archimedean then $L^{*}=L^{\wedge}$.

It is easy to see that if the Riesz space $E$ is intermediate with respect to the Riesz space $L$ then $E^{*}$ is an intermediate completion for $L$ and hence $E^{*}=L^{*}$. The following is an immediate consequence of this fact and Proposition 1.3.

Corollary 1.5. Let $L$ be a Riesz space. The Riesz space $E$ is intermediate with respect to $L$ if and only if $E$ is Riesz isomorphic to a Riesz subspace $E^{\prime}$ of $L^{*}$ such that $L \subseteq E^{\prime} \subseteq L^{*}$.

Thus, intermediate Riesz spaces can be thought of as spaces which "lie between" a given Riesz space and its intermediate completion.

2. Some specific intermediate spaces. There are three specific intermediate Riesz spaces in addition to the intermediate completion already discussed which we need to consider. The first of these we will call the intermediate- $\sigma$-completion.

Definition 2.1. Let $L$ be a Riesz space. The Riesz space $K$ will be called an intermediate- $\sigma$-completion of $L$ if the following conditions are satisfied.

(i) $K$ is intermediate with respect to $L$.

(ii) If $A$ is any bounded above countable subset of $K$ and $U$ is the set of all upper bounds for $A$ then $\sup A$ exists in $K$ if and only if $\inf \{u-a: u \in U$ and $a \in A\}=0$.

(iii) There exists an intermediate embedding $\phi$ of $L$ into $K$ such 
that if $E$ is any subspace of $K$ which satisfies (ii) and contains $\phi(L)$ then $E=K$.

THEOREM 2.2. Every Riesz space L has a unique up to lattice isomorphism intermediate- $\sigma$-completion.

Proof. We first note that $L^{*}$ is a Riesz space which satisfies conditions (i) and (ii) of Definition 2.1. Let $\phi$ be an intermediate embedding of $L$ into $L^{*}$ and let $K$ be the intersection of all Riesz subspaces of $L^{*}$ which contain $\phi(L)$ and satisfy condition (ii) of Definition 2.1. Then $K$ clearly satisfies all the conditions of Definition 2.1. Thus, intermediate- $\sigma$-completions exist. Now, suppose that $K_{1}$ and $K_{2}$ are intermediate- $\sigma$-completions of $L$ and suppose that $\pi_{1}$ and $\pi_{2}$ are intermediate embeddings of $L$ into $K_{1}$ and $K_{2}$, respectively, such that condition (iii) of Definition 2.1 is satisfied. Let $\pi_{1}^{\prime}$ and $\pi_{2}^{\prime}$ be intermediate embeddings of $K_{1}$ (respectively, $K_{2}$ ) into $K_{1}^{*}$ (respectively, $\left.K_{2}^{*}\right)$. Let $\phi_{1}=\pi_{1}^{\prime} \circ \pi_{1}$ and $\phi_{2}=\pi_{2}^{\prime} \circ \pi_{2}$. Since $K_{1}^{*}=K_{2}^{*}=L^{*}$, we can apply Proposition 1.3 to obtain an isomorphism of $K_{1}^{*}$ onto $K_{2}^{*}$ such that $\psi / \phi_{1}(L)=\phi_{2} \circ \phi_{1}^{-1}$. It follows easily from the fact that condition (iii) is satisfied for $\pi_{1}$ and $\pi_{2}$ that

$$
\psi\left(\pi_{1}^{\prime}\left(K_{1}\right)\right) \supseteq \pi_{2}^{\prime}\left(K_{2}\right)
$$

and

$$
\psi^{-1}\left(\pi_{2}^{\prime}\left(K_{2}\right)\right) \supseteq \pi_{1}^{\prime}\left(K_{1}\right)
$$

Hence, $\psi\left(\pi_{1}^{\prime}\left(K_{1}\right)\right)=\pi_{2}^{\prime}\left(K_{2}\right)$. So, $\pi_{2}^{\prime-1} \circ \psi \circ \pi_{1}^{\prime}$ is a Riesz isomorphism of $K_{1}$ onto $K_{2}$. The theorem is proved.

Condition (ii) of Definition 2.1 will reduce to $K$ being Dedekind- $\sigma$ complete in the event that $L$ is Archimedean.

Definition 2.3. Let $L$ be an Archimedian Riesz space. The Dedekind- $\sigma$-complete Riesz space $K$ is said to be a Dedekind- $\sigma$ completion for $L$ if the following conditions are satisfied:

(i) $K$ is intermediate with respect to $L$.

(ii) There exists an intermediate embedding $\phi$ of $L$ into $K$ such that if $\phi(L) \subseteq E$ is a Dedekind- $\sigma$-complete Riesz subspace of $K$ then $E=K$.

COROllary 2.4. Every Archimedean Riesz space L has a unique up to lattice isomorphism Dedekind-o-completion.

The next intermediate Riesz space we wish to consider is the order Cauchy completion. 
Definition 2.5. Let $L$ be a Riesz space. The sequence $\left\{x_{n}\right\}$ in $L$ is said to be order cauchy if there exists a sequence $\left\{z_{n}\right\} \subset L^{+}$with $z_{n} \downarrow 0$ such that, whenever $m \geqq n,\left|x_{n}-x_{m}\right| \leqq z_{n}$ where $m, n=1,2, \cdots$, .

Definition 2.6. The Riesz space $L$ is said to be order cauchy complete if every order cauchy sequence in $L$ order converges to an element of $L$.

The concept of an order cauchy sequence was first introduced in [5] by C. J. Everett (he called this concept $o$-regular). His work was later completed and extended by F. Papangelou in [14]. This next result is a consequence of [14, Lemma 2.10, p. 86].

Proposition 2.7. Let $L$ be a Riesz space. The sequence $\left\{x_{n}\right\}$ in $L$ is order cauchy if and only if there exist sequences $\left\{x_{n}^{\prime}\right\}$ and $\left\{x_{n}^{\prime \prime}\right\}$ in L with $x_{n}^{\prime} \downarrow, x_{n}^{\prime \prime} \uparrow, x_{n}^{\prime}-x_{n}^{\prime \prime} \downarrow 0$ and $x_{n}^{\prime \prime} \leqq x_{n} \leqq x_{n}^{\prime}$ for $n=1,2, \cdots$, .

We will take the following as our definition of an order cauchy completion.

Definition 2.8. Let $L$ be a Riesz space. The order cauchy complete Riesz space $K$ will be called an order cauchy completion of $L$ if the follwing conditions hold.

(i) $K$ is intermediate with respect to $L$.

(ii) There exists an intermediate embedding $\phi$ of $L$ into $K$ such that if $\phi(L) \subseteq E$ is an order cauchy complete Riesz subspace of $K$ then $E=K$.

It follows readily from Proposition 2.7 that the intermediate completion and the intermediate- $\sigma$-completion of a Riesz space $L$ are order cauchy complete. Using exactly the same techniques as were used in the proof of Theorem 2.2, we can now establish the following:

THEOREM 2.9. Every Riesz space L has a unique up to lattice isomorphism order cauchy completion.

Finally, we wish to discuss the concept of a relatively uniform completion.

Definition 2.10. Let $L$ be a Riesz space. The sequence $\left\{x_{n}\right\}$ in $L$ is said to be relatively uniform cauchy if there exist an element $x \in L^{+}$and a sequence $\left\{\lambda_{n}\right\}$ of real numbers with $\lambda_{n} \downarrow 0$ such that $\lambda_{n} x \downarrow 0$ and, for $m \geqq n,\left|x_{n}-x_{n}\right| \leqq \lambda_{n} x$ where $m, n=1,2, \cdots$, . 
Definition 2.11. The Riesz space $L$ is said to be relatively uniform complete if every relatively uniform cauchy sequence in $L$ has an order limit in $L$.

It is clear that any order cauchy complete Riesz space is relatively uniform complete. Thus, the intermediate completion, the intermediate- $\sigma$-completion, and the order cauchy completion of a Riesz space are all relatively uniform complete.

Definition 2.12. Let $L$ be a Riesz space. The relatively uniform complete Riesz space $K$ is said to be a relatively uniform completion for $L$ if the following conditions are satisfied.

(i) $K$ is intermediate with respect to $L$.

(ii) There exists an intermediate embedding $\phi$ of $L$ into $K$ such that if $\phi(L) \subseteq E$ is a relatively uniform complete Riesz subspace of $K$ then $E=K$.

Again, using exactly the same techniques as were used in the proof of Theorem 2.2, we can establish the following result.

THEOREM 2.13. Every Riesz space L has a unique up to lattice isomorphism relatively uniform completion.

In the sequel, if $L$ is a Riesz space, we will denote the intermediate$\sigma$-completion of $L$ by $L^{\prime}$, the order cauchy completion of $L$ by $L_{o}^{-}$, and the relatively uniform completion of $L$ by $L_{r u}^{-}$. We will continue to denote the intermediate completion of $L$ by $L^{*}$ and the Dedekind completion of an Archimedean Riesz space $L$ by $L^{\wedge}$. The notation $L^{\prime}$ will be used as well to denote the Dedekind- $\sigma$-completion of $L$ if $L$ is Archimedean. It is clear from the development of this section that $L_{r u}^{-}$ is intermediate with respect to $L, L_{o c}^{-}$is intermediate with respect to both $L_{r u}^{-}$and $L, L^{\prime}$ is intermediate with respect to $L_{o c}^{-}, L_{r u}^{-}$and $L$, and $L^{*}$ is intermediate with respect to all of the forementioned spaces.

3. Spaces which lie between two Riesz spaces. We begin this section by establishing a technical lemma which we need.

Lemma 3.1. Let $E$ be a Riesz space and $D \subseteq E^{+}$. Then $D$ is the generating positive cone for a Riesz subspace $D^{\prime}=D-D$ of $E$ if and only if $D$ satisfies the following conditions:

Let $x_{1}, x_{2} \in D$
(a) $x_{1}+x_{2} \in D$,
(b) $x_{1} \leqq x_{2}$ then $x_{2}-x_{1} \in D$,
(c) $0 \in D$, 
(d) either $x_{1} \vee x_{2}$ or $x_{1} \wedge x_{2}$ is in $D$,

(e) if $\alpha>0$ is a real number then $\alpha x_{1} \in E$.

Before proving this lemma, we note that the standard lattice identity, $x+y=x \vee y+x \wedge y$, combined with conditions (a) and (b) implies that if either $x_{1} \vee x_{2}$ or $x_{1} \wedge x_{2}$ is in $D$ then both are in $D$.

Proof. From conditions (a), (c) and (e) it follows that $D^{\prime}$ is a subspace of $E$. It is not difficult to show that the addition of condition (b) enables us to establish that the partial order on $D^{\prime}$ induced by $D$ coincides with that inherited from $E$ (i.e., $E^{+} \cap D^{\prime}=D$ ). To establish that $D^{\prime}$ is a Riesz subspace of $E$ with positive generating cone $D$ we need now only show that if $x \in D^{\prime}$ then $x^{+}=(x \vee 0)$ (in $\left.E\right)$ is in $D$ and combine this with what we have already pointed out (this suffices by virtue of [11, Th. 11.5(v) p. 56]). To this end, let $x=y_{1}-y_{2}$ be in $D^{\prime}$ where $y_{1}, y_{2} \in D$. It follows from [11, Th. 11.8, p. 58] that $x^{+}=$ $\left(y_{1}-y_{2}\right)^{+}=y_{1} \vee y_{2}-y_{2}$ (in $E$ ). By conditions (d) and (b) we have $y_{1} \vee y_{2}-y_{2} \in D$. The lemma is proved.

Throughout the remainder of this section $L$ will denote a Riesz space and $E$ will denote a Riesz space which is intermediate with respect to $L$. For convenience, we will assume that $L$ is a Riesz subspace of $E$ such that for each $e \in E$ we have

$$
e=\inf \{x \in L: x \geqq e\}=\sup \{y \in L: y \leqq e\} .
$$

We make the following definitions:

Definitions 3.2. (i) We will say that an element $e \in E^{+}$is a lower element if there exists a sequence $\left\{x_{n}\right\} \subset L$ such that $x_{n} \downarrow e$ (in $E$ ).

(ii) We will say that an element $e \in E^{+}$is an upper element if there exists a sequence $\left\{x_{n}\right\} \subset L^{+}$such that $x_{n} \uparrow e$ (in $E$ ).

(iii) We will say that an element $e \in E^{+}$is sequentially reachable from $L$ if there exists a sequence $\left\{x_{n}\right\} \subset L^{+}$such that $x_{n} \rightarrow e$ (in $E$ ).

(iv) We will say that an element $e \in E^{+}$is sequentially symmetric with respect to $L$ if it is both an upper element and a lower element.

Let $l(L, E), u(L, E), s(L, E)$, and $\sigma(L, E)$ denote, respectively, the set of all lower elements, upper elements, sequentially reachable elements, forementioned sequentially symmetric elements in $E$.

Consider, for the time being, the subspace of $E$ generated by $u(L, E)$ (i.e., $u(L, E)-u(L, E))$. First of all we show that $l(L, E) \subset$ $u(L, E)-u(L, E)$. To this end, let $e \in l(L, E)$. Then there exists a sequence $\left\{x_{n}\right\} \subset L^{+}$such that $x_{n} \downarrow e$ (in $E$ ) for $n=1,2, \cdots$, . Let $y_{n}=x_{1}-x_{n}$, for $n=1,2,3, \cdots$, . Then $0 \leqq y_{n} \uparrow x_{1}-e$ which implies that $x_{1}-e \in u(L, E)$ and hence that $e \in u(L, E)-u(L, E)$. 
We clearly have that if $x, y \in u(L, E)$ then $x \vee y \in u(L, E)$. We wish to show now that $u(L, E)-u(L, E)$ is a Riesz subspace of $E$ under the partial ordering induced by $E$. To see this all we need to note is that if $D=E^{+} \cap[u(L, E)-u(L, E)]$ then $D-D=u(L, E)-u(L, E)$ and $D$ satisfies all the conditions of Lemma 3.1 .

We now wish to establish that $l(L, E)-l(L, E)=$ $u(L, E)-u(L, E)$. We have already seen that $l(L, E)-l(L, E) \subseteq$ $u(L, E)-u(L, E)$. Let $x \in u(L, E)$. Then there exists a sequence $\left\{x_{n}\right\} \subset L^{+}$such that $x_{n} \uparrow x$ for $n=1,2, \cdots$, . Since $x \in E^{+}$, there exists a $y \in L^{+}$such that $y \geqq x$. If $y=x$ we are done. Assume, therefore, that $y>x$. Then, $\left\{y-x_{n}\right\} \subset L^{+}$and $y-x_{n} \downarrow y-x$ for $n=$ $1,2, \cdots$, . Thus, $y-x \in l(L, E)$ which implies that $x \in l(L, E)$. $l(L, E)$.

We have established the following therem.

THEOREM 3.3. For any $L$ and $E, u(L, E)-u(L, E)=$ $l(L, E)-l(L, E)$. Furthermore, $u(L, E)-u(L, E)$ is a Riesz subspace of $E$.

Remark 3.4. Since $\sigma(L, E)=u(L, E) \cap l(L, E)$, it is easy to see that the following held:

(i) If $l(L, E) \subseteq u(L, E)$, then $\sigma(L, E)=l(L, E)$.

(ii) If $u(L, E) \subseteq l(L, E)$, then $\sigma(L, E)=u(L, E)$.

(iii) If $u(L, E)=l(L, E)$, then $\sigma(L, E)=u(L, E)=l(L, E)$.

Theorem 3.5. Let $L_{\sigma(L . E)}=\sigma(L, E)-\sigma(L, E)$. Then $L_{\sigma(L . E)}$ is a Riesz subspace of $E$ with generating positive cone $\sigma(L, E)$; and, if $x_{n} \rightarrow x$ (in $L_{\sigma(L . E)}$ ) for $n=1,2, \cdots$, there exists a sequence $\left\{y_{n}\right\} \subset L^{+}$with $y_{n} \downarrow 0$ such that $\left|x-x_{n}\right| \leqq y_{n}$ for $n=1,2, \cdots$, .

Proof. To show that $L_{\sigma(L, E)}$ is a Riesz subspace of $E$ with generating positive cone $\sigma(L, E)$ we need only show that $\sigma(L, E)$ satisfies conditions (a), (b), (c), (d) and (e) of Lemma 3.1. Conditions (a), (c), (d) and (e) are all obvious, hence, all we need to show is condition (b). To this end, let $x_{1}, x_{2} \in \sigma(L, E)$ with $x_{1} \leqq x_{2}$. Let $\left\{x_{n}^{1}\right\}$ and $\left\{x_{n}^{2}\right\}$ be sequences in $L^{+}$such that $x_{n}^{2} \downarrow x_{2}$ (in $E$ ) and $x_{n}^{1} \uparrow x_{1}$ (in $E$ ) for $n=1,2, \cdots$, . Then, $x_{n}^{2}-x_{n}^{1} \downarrow x_{2}-x_{1}$ for $n=1,2, \cdots$, . Similarly, let $\left\{y_{n}^{1}\right\}$ and $\left\{y_{n}^{2}\right\}$ be sequences in $L^{+}$such that $y_{n}^{1} \downarrow x_{1}$ and $y_{n}^{2} \uparrow x_{2}$ for $n=1,2, \cdots$, . Then, $\left(y_{n}^{2}-y_{n}^{1}\right)^{+} \uparrow x_{2}-x_{1}$. This says that $x_{2}-x_{1}$ is both an upper and a lower element and hence is in $\sigma(L, E)$. Thus condition (b) of Lemma 3.1 is satisfied by $\sigma(L, E)$ and we have established that $L_{\sigma(L, E)}=\sigma(L, E)-\sigma(L, E)$ is a Riesz subspace of $E$ with generating positive cone $\sigma(L, E)$. 
For the remainder of the proof we need only show that if $\left\{x_{n}\right\}$ is any sequence in $\sigma(L, E)=L_{\sigma(L, E)}^{+}$such that $x_{n} \downarrow 0$ for $n=1,2, \cdots$, then there exists a sequence $\left\{y_{n}\right\} \subset L^{+}$with $y_{n} \geqq x_{n}$ and $y_{n} \downarrow 0$ for $n=$ $1,2, \cdots$, . Since $\left\{x_{n}\right\} \subset \sigma(L, E)$, for each $n$, there exists a sequence $\left\{z_{n, m}, m=1,2, \cdots,\right\} \subset L^{+}$such that $z_{n, m} \downarrow_{m} x_{n}$. Letting $y_{n}=\inf _{1 \leqq i \leqq n}\left\{z_{i, n}\right\}$ for $n=1,2, \cdots$, it is routine to show that $y_{n} \downarrow 0$ and satisfies the desired conditions. The theorem is proved.

The next theorem provides us with some further insight into the relationships between the sets $u(L, E)$ and $l(L, E)$.

THEOREM 3.6. If either $u(L, E)$ or $l(L, E)$ is the generating positive cone of a Riesz subspace of $E$, then both $u(L, E)$ and $l(L, E)$ are generating positive cones and in fact $u(L, E)=l(L, E)=\sigma(L, E)$.

Proof. Suppose that $u(L, E)$ is a generating positive cone for $u(L, E)-u(L, E)$. By virtue of Theorem 3.3 , we have $l(L, E) \subseteq$ $[u(L, E)-u(L, E)]^{+}=u(L, E)$ and by Remark 3.4 this implies that $l(L, E)=\sigma(L, E)$. Hence, $l(L, E)$ is the generating positive cone of the Riesz subspace $L_{\sigma(L . E)}$ of $E$. Since $l(L, E)-l(L, E)=$ $u(L, E)-u(L, E)$, we see that $\sigma(L, E)=l(L, E)=u(L, E)$. The case when $l(L, E)$ is the generating positive cone for a Riesz subspace of $E$ follows in exactly the same manner by interchanging the roles of $u(L, E)$ and $l(L, E)$ in the above argument. The theorem is proved.

We have established thus far that there are Riesz subspaces of $E$ which are naturally associated with the sets $\sigma(L, E), u(L, E)$ and $l(L, E)$ (namely, $L_{\sigma(L . E)}=\sigma(L, E)-\sigma(L, E)$ and $u(L, E)-u(L, E)=$ $l(L, E)-l(L, E))$. It is evident that the same is true for the set $s(L, E)$ since it is clearly a generating positive cone for a Riesz subspace of $E$. From now on we will denote $u(L, E)-u(L, E)$ by $L_{u(L, E)}$ and $s(L, E)-s(L, E)$ by $L_{s(L, E)}$. We will of course continue to denote $\sigma(L, E)-\sigma(L, E)$ by $L_{\sigma(L, E)}$.

Since $L_{\sigma(L . E)}, L_{u(L . E)}$ and $L_{s(L . E)}$ are again Riesz spaces and since $E$ is again intermediate with respect to each of these spaces, we can consider such spaces as

$$
\begin{aligned}
& L_{\sigma^{2}(L, E)}=\left(L_{\sigma(L, E)}\right)_{\sigma\left(L_{\sigma(L, F) . E)},\right.}, \\
& L_{u \sigma(L, E)}=\left(L_{\sigma(L, E)}\right)_{u\left(L_{\sigma r(L . K), E)},\right.}, \\
& L_{s^{2}(L, E)}=\left(L_{s(L, E)}\right)_{s\left(L_{s(L, F), E)}\right.},
\end{aligned}
$$

etc. For the time being we will not concern ourselves with any of the "higher order" spaces except those obtained by repeated application of the " $s$ " operation. 
To be more precise, let $\alpha$ be a countable ordinal. We define the family $\left\{L_{s^{\alpha}(L, E)}\right\}_{1 \leqq \alpha<\Omega}(\Omega=$ first uncountable ordinal) by

(i) $L_{s 1(L, E)}=L_{s(L, E)}$;

(ii) $L_{s^{\alpha}(L, E)}=\bigcup_{\beta<\alpha} L_{s^{\beta}(L, E)}$, for $\alpha$ a limit ordinal;

(iii) $L_{s^{\alpha+1}(L, E)}=\left(L_{s^{\alpha}(L, E)}\right)_{s\left(L s^{\alpha}(L, E), E\right)}$.

REMARK 3.7. If $\operatorname{cl}(L, E)$ denotes the closure of $L$ in the order topology on $E$, it is easily seen that, for each $1 \leqq \alpha<\Omega, L_{s^{\alpha}(L . E)} \subseteq$ $\operatorname{cl}(L, E)$ (just note first that the " $s$ " operation corresponds to taking pseudo order closures and use the theory in [11, p. 82]). Furthermore, a standard argument of set theory will establish that $\operatorname{cl}(L, E)=$ $\cup_{1 \leqslant \alpha<\Omega} L_{s^{\alpha}(L, E)}$. In Particular, this implies that $\operatorname{cl}(L, E)$ is a Riesz subspace of $E$.

At this point we would like to introduce one additional class of spaces.

Definition 3.8. Let $L$ be a Riesz space and let $E$ be a Riesz space which is intermediate with respect to $L$. The element $e \in E^{+}$will be called a relatively uniform reachable element of $E$ if there exist a sequence $\left\{x_{n}\right\} \subset L^{+}$, a sequence $\left\{\lambda_{n}\right\} \subset R^{+}$and an $x \in L^{+}$such that $\lambda_{n} x \downarrow 0$ and, for each $n=1,2, \cdots,\left|x_{n}-e\right| \leqq \lambda_{n} x$.

We will denote by $r u(L, E)$ the set of all elements of $E^{+}$which are relatively uniform reachable from $L$.

Remark 3.9. For any $L$ and $E, r u(L, E) \subseteq \sigma(L, E)$. To see this, let $e \in E^{+}$and let $\left\{x_{n}\right\}$ and $\left\{\lambda_{n}\right\}$ be sequences in $L^{+}$and $R^{+}$, respectively, such that $\left|x_{n}-e\right| \leqq \lambda_{n} x \downarrow 0$ for some $x \in L^{+}$. Then, for each $n,-\lambda_{n} x$ $\leqq e-x_{n} \leqq \lambda_{n} x$ and hence $x_{n}-\lambda_{n} x \leqq e \leqq x_{n}+\lambda_{n} x$. For each $n$, let $z_{n}^{\prime}$ and $z_{n}^{\prime \prime}$ be defined by $z_{n}^{\prime}=\inf _{1 \leqq i \leqq n}\left\{x_{i}+\lambda_{i} x\right\}$ and $z_{n}^{\prime \prime}=$ $\sup _{1 \leqq i \leqq n}\left\{\left(x_{i}-\lambda_{i} x\right)^{+}\right\}$. Then $\left\{z_{n}^{\prime}\right\}$ and $\left\{z_{n}^{\prime \prime}\right\}$ are sequences in $L^{+}$such that $z_{n}^{\prime} \downarrow e$ and $z_{n}^{\prime \prime} \uparrow e$. Hence, $e \in \sigma(L, E)$.

It is clear that $r u(L, E)$ is the generating positive cone for a Riesz subspace of $E$. We let $L_{r u(L . E)}=r u(L, E)-r u(L, E)$. For each countable ordinal $\alpha$, one defines the spaces $L_{(r u)^{\alpha}(L, E)}$ in the same way that the spaces $L_{s^{\alpha}(L . E)}$ were previously defined.

LEMMA 3.10. For any $L$ and $E, L_{\sigma^{2}(L . E)}=L_{\sigma(L . E)}$.

Proof. Let $e \in E$ be in $\sigma\left(L_{\sigma(L . E)}, E\right)$. Then, there exist sequences $\left\{x_{n}^{\prime}\right\}$ and $\left\{x_{n}^{\prime \prime}\right\}$ in $\sigma(L, E)$ such that $x_{n}^{\prime} \downarrow e$ and $x_{n}^{\prime \prime} \uparrow e$. Since $x_{n}^{\prime} \in$ $\sigma(L, E)$ there exists, for each $n$, a sequence $\left\{x_{n, m}^{\prime}\right\}$ in $L^{+}$such that $x_{n, m}^{\prime} \downarrow_{m} x_{n}^{\prime}$. Similarly, for each $n$, there exists a sequence $\left\{x_{n, m}^{\prime \prime}\right\}$ in $L^{+}$ such that $x_{n, m}^{\prime \prime} \uparrow_{m} x_{n}^{\prime \prime}$. Let, for each $n, z_{n}^{\prime}$ and $z_{n}^{\prime \prime}$ be defined by $z_{n}^{\prime}=$ 
$\inf _{1 \leqq i \leqq n}\left\{x_{i, n}^{\prime}\right\}$ and $z_{n}^{\prime \prime}=\sup _{1 \leqq i \leqq n}\left\{x_{i, n}^{\prime \prime}\right\}$. Then, $\left\{z_{n}^{\prime}\right\}$ and $\left\{z_{n}^{\prime \prime}\right\}$ are sequences in $L^{+}$such that $z_{n}^{\prime} \downarrow e$ and $z_{n}^{\prime \prime} \uparrow e$. We have shown that $L_{\sigma^{2}(L . E) \subseteq}$ $L_{\sigma(L . E)}$. Since showing the other containment is trivial, the assertion is proved.

REmark 3.11. It follows easily from Lemma 3.10 that if $L \subseteq L_{1} \subseteq$ $L_{\sigma(L, E)}$ then $L_{\mid \sigma\left(L_{1}, E\right)}=L_{\sigma(L, E)}$. Hence, for every $1 \leqq \alpha<\Omega$, we have that $L_{(r u)^{\alpha}(L, E)} \subseteq L_{\sigma(L, E)}$. If $\operatorname{cl}^{\prime}(L, E)$ denotes the relatively uniform closure of $L$ in $E$ (see [11, pp. 84-86]) then it can be established that $\operatorname{cl}^{\prime}(L, E)=\bigcup_{1 \leqq \alpha<\Omega} L_{(r u)^{\alpha}(L, E)}$ and hence that $\operatorname{cl}^{\prime}(L, E) \subseteq L_{\sigma(L, E)}$ and is a Riesz subspace of $E$.

We say that a Riesz space $L$ is order separable if every nonempty subset $D$ possessing a supremum in $L$ contains an at most countable subset possessing the same supremum in $L$ as does $D$.

Lemma 3.12. Let L be a Riesz space and let E be a Riesz space which is intermediate with respect to $L$. Then $L$ is order separable if and only if $E$ is order separable.

Proof. It is easy to see that if $E$ is order separable then $L$ is order separable. Thus we are left with showing that if $L$ is order separable then $E$ is order separable. It follows from [11, Th. 23.2 (iii), p. 125] that a Riesz space $L$ is order separable if and only if whenever $\left\{x_{\alpha}\right\}$ is a net in $L$ with $x_{\alpha} \downarrow 0$ there exists a sequence $\left\{x_{\alpha_{n}}\right\} \subseteq\left\{x_{\alpha}\right\}$ such that $x_{\alpha_{n}} \downarrow 0$. Let $\left\{x_{\alpha}\right\}$ be a net in $E$ such that $x_{\alpha} \downarrow 0$. Let $V=$ $\left\{y \in L: y \geqq x_{\alpha}\right.$ for some $\left.\alpha\right\}$. Clearly inf $V=0$ (since $E$ is intermediate with respect to $L$ ). Since $L$ is order separable, there exists $\left\{y_{n}\right\} \subset V$, for $n=1,2, \cdots$, such that $\inf \left\{y_{n}\right\}=0$. Let $\left\{\alpha_{n}\right\} \subseteq\{\alpha\}$ be chosen so that, for each $n, x_{\alpha_{n+1}} \leqq x_{\alpha_{n}}$ and $x_{\alpha_{n}} \leqq y_{n}$. Then $x_{\alpha_{n}} \downarrow 0$ and $\left\{x_{\alpha_{n}}\right\} \subseteq$ $\left\{x_{\alpha}\right\}$. Hence, $E$ is order separable. The assertion is proved.

COROLlaRY 3.13. Let L be an order separable Riesz space and let $E$ be any Riesz space which is intermediate with respect to $L$. Then $L_{\sigma(L . E)}=E$.

Proof. Without loss of generality, we assume that $L$ is a Riesz subspace of $E$ such that for every $e \in E$ we have that $e=$ $\sup \{x \in L: x \leqq e\}=\inf \{y \in L: y \geqq e\}$. By virtue of Lemma 3.8, it follows that $E$ is order separable and hence that, for any $e \in E$, we have sequences $\left\{x_{n}\right\},\left\{y_{n}\right\} \subset L$ such that $x_{n} \uparrow e$ and $y_{n} \downarrow e$. But then each element of $E^{+}$is in $\sigma(L, E)$. We have established that $L_{\sigma(L, E)}=E$. The assertion is proved.

4. Spaces between $L$ and $L^{*}$. In this section the investigations begun in the preceding section are continued for the special case 
when the intermediate space $E$ is taken to be $L^{*}$ ( $L$ 's intermediate completion). Again we assume that $L$ is a Riesz subspace of $L^{*}$ such that for each $\hat{x} \in L^{*}$,

$$
\hat{x}=\sup \{x \in L: x \leqq x\}=\inf \{y \in L: y \geqq \hat{x}\}
$$

We will simplify the notation of the previous section by denoting $\sigma\left(L, L^{*}\right), \quad l\left(L, L^{*}\right), \quad u\left(L, L^{*}\right), \quad r u\left(L, L^{*}\right), \quad s\left(L, L^{*}\right), \quad L_{\sigma\left(L . L_{1}^{*}\right)}, \quad L_{u\left(L . L_{*}^{*}\right)}$, $L_{(r u)^{\alpha}\left(L, L^{*}\right)}$ and $L_{s^{\alpha}\left(L, L^{*}\right)}($ where $1 \leqq \alpha<\Omega)$ by $\sigma(L), l(L), u(L), r u(L)$, $s(L), L_{\sigma}, L_{u}, L_{(r u)^{\alpha}}$ and $L_{s}{ }^{\alpha}$, respectively.

THEOREM 4.1. Let $L$ be a Riesz space. The following statements are valid:

(i) $\quad L_{o c}^{-}=L_{\sigma}$

(ii) If $x_{n} \downarrow 0$ in $L_{o c}^{-}$, there exists $y_{n} \downarrow 0$ in $L$ such that, for each $n=1,2, \cdots, x_{n} \leqq y_{n}$.

(iii) $L$ is order separable if and only if $L_{o c}^{-}$is order separable.

(iv) If $L$ is order separable, then $L_{o c}^{-}=L^{*}$.

(v) If $L$ is an Archimedean order separable Riesz space, then $L_{\text {or }}^{-}=L^{\wedge}$.

(vi) $L_{r u}^{-}=\operatorname{cl}^{\prime}(L)=\bigcup_{1 \leqq \alpha<\Omega} L_{(r u)^{\alpha}}$.

(vii) $L^{\prime}=\operatorname{cl}(L)=\cup_{1 \leqq \alpha<\Omega} L_{s}$.

Proof. It is not difficult to establish, using Proposition 2.7, that no proper Riesz subspace of $L_{\sigma}$ containing $L$ can be order cauchy complete. That $L_{\sigma}$ is order cauchy complete is a consequence of Proposition 2.7 and Lemma 3.10. Thus, $L_{\sigma}$ satisfies the conditions of Definition 2.8. The validity of (i) now follows from Theorem 2.9.

The validity of (ii) follows from (i) and Theorem 3.5.

The validity of (iii) follows from Lemma 3.12.

That (iv) is valid is a consequence of (i) and Corollary 3.13.

That ( $v$ ) is valid is a consequence of (iv) and of the fact that in this case $L^{\wedge}=L^{*}$.

The validity of (vi) is an easy consequence of Theorem 2.13.

That (vii) is true follows from Theorem 2.2.

REMARK 4.2. It follows from (iii) and (v) of Theorem 4.1 that, for Archimedean Riesz spaces, $L$ is order separable if and only if $L^{\wedge}$ is order separable (see [11, Th. 32.9, p. 196]). Assertions (i), (ii) and (vii) of Theorem 4.1 could have been obtained in other ways from results in [14].

We have the following containment schemes:

REMARK 4.3. If $L$ is a Riesz space, then 


$$
\begin{aligned}
& \begin{array}{clll}
L_{r u}^{-} & L_{o c}^{-} & L^{\prime} & L^{\prime} \\
\| & \| & \| & \|
\end{array} \\
& L \subseteq L_{(r u)^{\alpha}} \subseteq \operatorname{cl}^{\prime}(L) \subseteq L_{\sigma} \subseteq L_{u} \subseteq L_{s^{\beta}} \subseteq \operatorname{cl}(L) \subseteq L^{*} \text {, }
\end{aligned}
$$

and

$$
\begin{aligned}
& L^{+} \subseteq(r u)^{\alpha}(L) \subseteq \sigma(L) \subseteq^{u(L)} \subseteq{ }_{s^{\beta}(L) \subseteq L^{\prime+} \subseteq L^{*+}} . \\
& \subseteq_{l(L)} \subseteq
\end{aligned}
$$

This next result provides us with some more information concerning the above containment schemes.

TheOREM 4.4. Let L be a Riesz space. Then the following are equivalent :

(i) $\quad u(L)$ is a generating positive cone for a Riesz subspace of $L^{*}$.

(ii) $\quad l(L)$ is a generating positive cone for a Riesz subspace of $L^{*}$.

(iii) $u(L)=l(L)=\sigma(L)$.

(iv) $L_{\sigma}=L^{\prime}$.

(v) $\quad l(L) \subseteq u(L)$.

(vi) $u(L) \subseteq l(L)$.

Proof. The implications (i) $\Rightarrow$ (ii) $\Rightarrow$ (iii) follow from Theorem 3.6. To see that (iv) $\Rightarrow$ (v) we use the containment scheme in Remark 4.3 to see that $l(L) \subseteq L^{\prime+}=\sigma(L) \subseteq u(L)$. The implications (v) $\Rightarrow$ (vi) and (vi) $\Rightarrow$ (i) follow by first applying Remark 3.4 and then Theorem 3.6. Thus all that remains to be shown is that (iii) $\Rightarrow$ (iv). To this end, let $\left\{x_{n}\right\} \subset L_{\sigma}^{+}=\sigma(L)$, for $n=1,2, \cdots$, be such that $\inf \left\{x_{n}-v: n=\right.$ $1,2, \cdots, v \in V\}=0$ (where $V$ is the set of all lower bounds for $\left\{x_{n}\right\}$ ). We may as well assume that $x_{n} \downarrow x \in L^{*+}$ since otherwise we can replace the sequence $\left\{x_{n}\right\}$ by the sequence $\left\{y_{n}\right\}$ where, for each $n=1,2, \cdots, y_{n}=\inf _{1 \leqq i \leqq n}\left\{x_{i}\right\}$. Since, for each $n, x_{n} \in \sigma(L)$ there exists a sequence $\left\{z_{n, m}, m=1,2, \cdots,\right\} \subset L^{+}$such that $z_{n, m} \downarrow_{m} x_{n}$. Taking $y_{n}=$ $\inf _{1 \leqq i \leqq n}\left\{z_{i, n}\right\}$, we obtain a sequence $\left\{y_{n}\right\}$ in $L^{+}$such that $y_{n} \downarrow x$, where $n=1,2, \cdots$, . This says that $x \in l(L)$, but $l(L)=\sigma(L)$ (by condition (iii)), hence $x \in \sigma(L)$. We have proved that (iii) $\Rightarrow$ (iv). The theorem is proved.

5. Some examples. In this section, we give some examples which show (with one exception) that the containment schemes in Remark 4.3. are best possible. The one exception is with respect to the containment $L_{(r u)^{\alpha}} \subseteq L_{r u}^{-}$. In our first example we show that there is an 
Archimedean Riesz space $L$ such that $L \subset L_{r u}=$ $L_{r u}^{-} \subset L_{\sigma}$. Unfortunately, we do not know whether it is always true that $L_{(r u)^{2}}=L_{r u}$ and hence that $L_{r u}=L_{r u}^{-}$. In other words, the consideration of the spaces $L_{(r u)^{\alpha}}$ for $\alpha \geqq 2$ may be superfluous. We will have a few more remarks to make about this situation a little later on.

Example 5.1. Let $L$ be the Archimedean Riesz space whose elements are the piece-wise polynomial functions defined on the unit interval. It is not difficult to see, using [11, Th. 32.6 p. 195], that $L^{\wedge}$ and $C([0,1])^{\wedge}$ (where $C([0,1])$ is the Riesz space of continuous, real valued functions on the unit interval) are the same. Hence, $C([0,1])$ is intermediate with respect to $L$. Clearly, $L_{r u}=L_{r u}^{-}=C([0,1])$. On the otherhand, it follows from Theorem 4.1 (i) and (v) together with the fact that $L$ and $C([0,1])$ are order separable that $L^{\wedge}=C([0,1])^{\wedge}=$ $L_{o c}^{-}=L_{\sigma}$. Since, as is well known, $C([0,1])$ is not Dedekind complete, we have that $L \neq L_{\text {ru }} \neq L_{\sigma}=L^{\wedge}$.

In view of Theorem 4.4, we see that the only possibility for nontrivial inclusions between $L_{\sigma}$ and $L^{\prime}$ in the inclusion scheme in Remark 4.3 occurs when $u(L) \notin \subset l(L)$ and $l(L) \notin u(L)$. In this next example, an Archimedean Riesz space $L$ is given such that $L \neq L_{\sigma} \neq L^{\prime}$.

ExAmple 5.2. Let $X$ be an uncountable cardinal and let $F(X)$ be the Riesz space of all bounded real valued functions on $X$. Let $L$ be the Riesz subspace of $F(X)$ consisting of all finitely nonconstant functions $f \in F(X)$. Clearly, $L^{\wedge}=F(X)$. We will show first that $l(L) \notin \subset u(L)$. It then follows from Theorem 4.4 that $u(L) \notin \subset l(L)$ and that $L_{\sigma} \neq L^{\prime}$. Let $\left\{x_{k} ; k=1,2, \cdots,\right\} \subset X$ be any countably infinite subset of $X$. Define the functions $f_{n}$ in $L^{+}$by:

$$
f_{n}(x)=\left\{\begin{array}{c}
0 ; \text { for } x=x_{h}, k=1,2, \cdots, n, \\
1 ; \text { otherwise }
\end{array}\right.
$$

where $n=1,2, \cdots$, . Then, $0 \leqq f_{n} \downarrow f\left(\right.$ in $\left.L^{\wedge}\right)$ where

$$
f(x)=\left\{\begin{array}{c}
0 ; \text { for } x=x_{k}, k=1,2, \cdots, \\
1 ; \text { otherwise }
\end{array}\right.
$$

If $g \in L$ is such that $0 \leqq g \leqq f$, then $g(x)=0$ except for at most a finite number of $x \in X$. Hence, there can be no sequence $\left\{g_{n}\right\}_{n=1}^{x} \subset L^{+}$ such that $g_{n} \uparrow f$ and thus $f \notin u(L)$. We have established that $l(L) \not \subset u(L), u(L) \not \subset l(L)$ and $L_{\sigma} \neq L^{\prime}$.

We will now show that $L_{\sigma} \neq L$. To this end, let $\left\{x_{k} ; k=1,2, \cdots,\right\}$ be a countably infinite point set in $X$. Define the functions $f_{n}$ by 


$$
f_{n}(x)=\left\{\begin{array}{c}
1 / k ; \text { for } k=1,2, \cdots, n, n=x_{k}, \\
0 ; \text { otherwise }
\end{array}\right.
$$

where $n=1,2, \cdots$, . Define the functions $g_{n}$ by

$$
g_{n}(x)=\left\{\begin{array}{l}
1 / k ; \text { for } x=x_{k}, k=1,2, \cdots, n, \\
1 / n ; \text { otherwise }
\end{array}\right.
$$

where $n=1,2, \cdots$, . Then, $\left\{f_{n}\right\},\left\{g_{n}\right\} \subset L^{+}$and $f_{n} \uparrow f, g_{n} \downarrow f$ where

$$
f(x)=\left\{\begin{array}{c}
1 / k ; x=x_{k}, k=1,2, \cdots \\
0 ; \text { otherwise }
\end{array}\right.
$$

Thus, $f \in \sigma(L)$ but $f \notin L$. We have shown that $L \neq L_{\sigma}$.

We will now find the Dedekind- $\sigma$-completion of $L$. Consider $D(X)=\{f \in F(X): f$ is countably non-constant $\}$. Obviously, $D(X)$ is Dedekind- $\sigma$-complete and $D(X) \supset L$. Let $f \in D(X)^{+}$and let $\left\{x_{k} ; k=1,2, \cdots,\right\}$ be the at most countable subset of $X$ off of which $f$ is constant. Suppose $\alpha \in R$ is such that $f(x)=\alpha$ for $x \in X-\left\{x_{k} ; k=1,2, \cdots,\right\}$. Let $\left\{x_{k_{n}} ; n=1,2, \cdots,\right\}$ be the subsequence of $\left\{x_{k} ; k=1,2, \cdots,\right\}$ such that $f\left(x_{k_{n}}\right)<\alpha$ for $n=1,2, \cdots$, . Let $\left\{x_{k_{p}} ; p=1,2, \cdots,\right\}=\left\{x_{k} ; k=1,2, \cdots\right\}-\left\{x_{k_{n}} ; n=1,2, \cdots,\right\}$ (we might as well assume that both of these sets are actually countably infinite). Define $f_{m}(x)$ by,

$$
f_{m}(x)=\left\{\begin{array}{c}
f\left(x_{k_{n}}\right) ; x=x_{k_{n}}, n=1,2, \cdots, m, \\
\alpha ; \text { otherwise }
\end{array}\right.
$$

where $m=1,2, \cdots$, . Define $g_{m}(x)$ by,

$$
g_{m}(x)=\left\{\begin{array}{c}
f\left(x_{k_{p}}\right)-\alpha ; \text { for } x=x_{k_{p}}, p=1,2, \cdots, m, \\
0 ; \text { otherwise }
\end{array}\right.
$$

where $m=1,2, \cdots$, . Now, $\left\{f_{m}\right\},\left\{g_{m}\right\} \subset L^{+}, g_{m} \uparrow(f-\alpha) \vee 0 \in u(L)$. $f_{m} \downarrow(f \wedge \alpha) \in l(L)$, and $((f-\alpha) \vee 0)+(f \wedge \alpha)=f$. Hence, $D(X)=L_{u}$ is the Dedekind- $\sigma$-completion of $L$ (i.e., $D(X)=L^{\prime}$ ).

It follows from our previous discussions that if $L$ is Archimedean and order separable then $L_{\sigma}=L^{\prime}=L_{o c}^{-}=L^{\wedge}$ (indeed, we used this fact in Example 5.1). The following is an example of a non-order separable non Dedekind- $\sigma$-complete Archimedean Riesz space $L$ such that $L_{\sigma}=L^{\prime}$.

Example 5.3. Let $F(X)$ and $D(X)$ be the Riesz spaces of Example 5.2. Let $L$ be the Riesz subspace of $D(X)$ consisting of all 
functions $f \in D(X)$ such that if $\alpha$ is that real number for which the cardinality of the set $\{x \in X: f(x)=\alpha\}$ is uncountable and if $\left\{x_{n}\right\}=$ $X-\{x \in X: f(x)=\alpha\}$, then the sequence $\left\{f\left(x_{n}\right): n=1,2, \cdots,\right\}$ has finitely many limit points. We want to show that $D(X)=L_{\sigma}$. It is sufficient to show that $D(X)^{+} \subseteq \sigma(L)$. To this end, let $f \in D(X)^{+}$and let $\alpha$ be that real number such that $\{x: f(x)=\alpha\}$ is uncountable. Let $\left\{x_{n}\right\}=X-\{x: f(x)=\alpha\}$ (we may assume $\left\{x_{n}\right\}$ is countably infinite since otherwise $f \in L)$. Let $M=\max \left\{f\left(x_{n}\right) ; n=1,2, \cdots,\right\}$. Define $u_{m}$ by

$$
u_{m}(x)=\left\{\begin{array}{c}
f\left(x_{n}\right) ; x=x_{n}, n=1,2, \cdots, m \\
M ; x=x_{n}, n=m+1, m+2, \cdots \\
\alpha ; \text { otherwise }
\end{array}\right.
$$

where $m=1,2, \cdots$, . Define $v_{m}$ by

$$
v_{m}(x)=\left\{\begin{array}{l}
f\left(x_{n}\right) ; x=x_{n}, n=1,2, \cdots, m \\
0 ; x=x_{n}, n=m+1, m+2, \cdots \\
\alpha ; \text { otherwise }
\end{array}\right.
$$

where $m=1,2, \cdots$, . We have that $\left\{u_{m}\right\},\left\{v_{m}\right\} \subset L^{+}$. It is clear that $u_{m} \downarrow f$ and $v_{m} \uparrow f$. This says that $f \in u(L) \cap l(L)=\sigma(L)$. Thus $L^{\prime}=$ $D(X)=L_{\sigma}$.

The reader should note that in both of the preceding examples $L^{\prime}=D(X)$ is not Dedekind complete.

We have remarked that for any Archimedean Riesz space $L, L^{\prime}=$ $\cup_{\alpha<\Omega} L_{s}{ }^{\alpha}$. In this next example, we show that there exists an Archimedean Riesz space $L$ such that, for each $\alpha<\Omega, L_{s^{\alpha}} \neq L_{s^{\alpha+1}}$. For the terminology and theory behind this example we refer the reader to [7, pp. 344-404].

Example 5.4. Let $X$ be the unit interval and let $F(X)$ be the Riesz space of all bounded real valued functions on $X$. For our Riesz space $L$, we will take the collection of all simple functions $f \in F(X)$ such that $f=\sum_{i=1}^{n} \alpha_{i} x_{A_{i}}$ where, for each $i, A_{i}$ is an ambiguous set of type 1 (i.e., $A_{i}$ is both $F_{\sigma}$ and $G_{\delta}$ ). Since characteristic functions of singleton sets and the function $\chi_{[0.1]}$ are all in $L$, it follows immediately from [11, Th. 32.6, p. 195] that $L^{\wedge}=F(X)$. By virtue of [7, Th's 1 and 2 p. 374; Th. 4, p. 389] we have that for each $\alpha<\Omega, L_{s^{\alpha}} \neq L_{s^{\alpha+1}}$. The Dedekind- $\sigma$-completion $L^{\prime}$ of $L$ is just the space of analytic representable functions (see the Lebesgue-Hausdorff Theorem in [7, p. 393]).

6. Extending order convergence properties. We begin by giving the definitions of some order convergence properties. We will number each property and in the sequel when we say a Riesz space 
$L$ has property $(r)$ where $r \in\{1,2,3,4,5\}$ we will be referring to these properties by number.

(1) The Riesz space $L$ is said to have the $\sigma$-property (property (1)) if for any sequence $\left\{u_{n}: n=1,2, \cdots,\right\}$ in $L^{+}$, there is a sequence $\left\{\lambda_{n}: n=1,2, \cdots,\right\}$ of positive real numbers and an element $u \in L^{+}$such that $u_{n} \leqq \lambda_{n} u$ for all $n$.

(2) The Riesz space $L$ is said to have property (2) if in $L$ order convergence for sequences and relatively uniform convergence are equivalent.

(3) Order convergence in the Riesz space $L$ is said to be stable ( $L$ is said to have property (3)) if for every sequence $\left\{x_{n}: n=1,2, \cdots,\right\}$ in $L$ with $x_{n} \rightarrow 0$ there exists a sequence of real numbers $\left\{\lambda_{n}: n=1,2, \cdots,\right\}$ such that $\lambda_{n} \uparrow \infty$ and $\lambda_{n} x_{n} \rightarrow 0$.

(4) The Riesz space $L$ is said to have the diagonal property (property (4)) if whenever $f_{n, k}, f_{n}, f$ in $L$ are such that $f_{n, k} \rightarrow{ }_{k} f_{n}$ for $n=1,2, \cdots$, and $f_{n} \rightarrow f$ there exists a "diagonal sequence" $\left\{f_{n, k(n)}\right\}$ such that $f_{n, k(n)} \rightarrow f$, where $n, k=1,2, \cdots$, .

(5) The Riesz space $L$ is said to have the Egoroff property (property (5)) if for every $f \in L$ and double sequence $\left\{0 \leqq b_{n, k}: n, k=\right.$ $1,2, \cdots$,$\} in L$ with $b_{n, k} \uparrow_{k}|f|$ there exists a sequence $0 \leqq b_{m} \uparrow|f|$ such that, for any $m, n$, there exists a $j(m, n)$ such that $b_{m} \leqq b_{n, j(m, n)}$.

These properties and several related properties are discussed in [16]. For a really complete discussion of these properties, their interrelationships, and their connections with other properties, the reader should see [11, Ch. 10].

We have the following lemma.

Lemma 6.1. Let L and E be Riesz spaces with E intermediate with respect to $L$. Then the following implications hold:

(i) $E$ has property (1) $\Leftrightarrow L$ has property (1).

(ii) E has property (2) $\Rightarrow L$ has property (2).

(iii) If $E$ is Archimedean and has property (3) then L has property (3). (4).

(iv) If $E$ is Archimedean and has property (4) then L has property

Proof. The proof of (i) is straightforward and is omitted. We begin by proving (ii). Suppose that $E$ has property (2) and let $\left\{x_{n}\right\}$ be a sequence in $L$ such that $x_{n} \rightarrow 0$ (in $L$ ). Then $x_{n} \rightarrow 0$ (in $E$ ) and since $E$ has property (2) there exist a sequence $\left\{\lambda_{n}\right\} \subset R^{+}$with $\lambda_{n} \downarrow 0$ and an $e \in E^{+}$such that $\left|x_{n}\right| \leqq \lambda_{n} e$. Since $E$ is intermediate with respect to $L$, there exists an $x \geqq e$ in $L$. But then $\lambda_{n} x \geqq \lambda_{n} e \geqq\left|x_{n}\right|$ for each $n$. Hence, if $x_{n} \rightarrow 0$ (in $L$ ) then $x_{n} \rightarrow 0(r \cdot u)$ (in $L$ ). To show that if $x_{n} \rightarrow 0(r \cdot u)($ in $L)$ then $x_{n} \rightarrow 0$ it suffices to show that if $x \in L^{+}$and $\left\{\lambda_{n}\right\}$ 
is a sequence in $R$ with $\lambda_{n} \downarrow 0$ then $\lambda_{n} x \downarrow 0$ (in $L$ ). But, since $E$ has property (2), $\lambda_{n} x \downarrow 0$ (in $E$ ) (i.e., $\lambda_{n} x \downarrow$ and $\inf \left\{\lambda_{n} x: n=1,2, \cdots,\right\}=0$ ) and hence $\lambda_{n} x \downarrow 0$ (in $L$ ).

The validity of (iii) follows from the fact that property (3) and property (2) are equivalent in Archimedean Riesz spaces (c.f., [11, Th. 16.3, p. 80]) and $L$ is Archimedean if $E$ is.

The validity of (iv) follows from the fact that property (1) together with property (3) is equivalent to property (4) in Archimedean Riesz spaces (c.f., [11, Th. 70.2, p. 478]).

The lemma is proved.

REMARK 6.2. The reader should note that if a Riesz space has property (2) then it must be Archimedean.

We will give some examples in $\S 7$, which show, first, that Archimedean is necessary for (iii) and (iv) of Lemma 6.1 to be valid; and, second, that the implications in parts (iii) and (iv) do not reverse even when $L$ is Archimedean.

Right now we will concern ourselves with the main theorem of this section.

THEOREM 6.3. Let L be a Riesz space and let E be a Riesz space intermediate with respect to $L$. Then the following implications are true:
(i)
L has property
$(2) \Leftrightarrow L_{\sigma(L, E)}$ has property (2).
(ii)
$(3) \Leftrightarrow L_{\sigma(L, E)}$ has property (3).
(iii) L has property
$(4) \Leftrightarrow L_{\sigma(L . E)}$ has property (4).
(iv)
$(5) \Leftrightarrow L_{\sigma(L . E)}$ has property (5).

Proof. We begin by establishing that (i) is valid. That $L_{\sigma(L . E)}$ has property (2) $\Rightarrow L$ has property (2) follows from Lemma 6.1 (ii). To see that $L$ has property (2) $\Rightarrow L_{\sigma(L . E)}$ has property (2), let $\left\{x_{n}: n=1,2, \cdots,\right\}$ be a sequence in $L_{\sigma(L . E)}$ such that $x_{n} \rightarrow x \in L_{\sigma(L . E)}$. By Theorem 3.5, there exists a sequence $\left\{y_{n}\right\}$ in $L$ with $y_{n} \downarrow 0$ such that $\left|x-x_{n}\right| \leqq y_{n}$ for $n=1,2, \cdots$, . Since $L$ has property (2), there exist a sequence $\left\{\lambda_{n}\right\}$ of real numbers with $\lambda_{n} \downarrow 0$ and a $y \in L^{+}$such that $y_{n} \leqq \lambda_{n} y$ for each n. But then $\left|x-x_{n}\right| \leqq y_{n} \leqq \lambda_{n} y$ for each $n$ and hence $x_{n} \rightarrow x(r \cdot u)$. The fact that if the sequence $\left\{x_{n}: n=1,2, \cdots,\right\}$ in $L_{\sigma(L . E)}$ is such that $x_{n} \rightarrow x(r \cdot u)$ where $x \in L_{\sigma(L . E)}$ implies $x_{n} \rightarrow x$ (in $L_{\sigma(L . E)}$ ) is an easy consequence of the facts that $L_{\sigma(L . E)}$ is intermediate with respect to $L$ and $L$ has this property. We have established (i).

We will now establish that (ii) is valid. First, assume that $L_{\sigma(L . E)}$ has property (3) and let $\left\{x_{n}: n=1,2, \cdots,\right\}$ be a sequence in $L$ such that $x_{n} \downarrow 0$ (in $L$ and, hence, in $\left.L_{\sigma(L . E)}\right)$. Since $L_{\sigma(L . E)}$ has property (3), there exists a sequence of real numbers $\left\{\lambda_{n}: n=1,2, \cdots,\right\}$ with $\lambda_{n} \uparrow \infty$ such 
that $\lambda_{n} x_{n} \rightarrow 0$ (in $L_{\sigma(L . E)}$ ). But then $\lambda_{n} x_{n} \rightarrow 0$ (in $L$ ) by virtue of Theorem 3.5. We have established that $L_{\sigma(L, E)}$ has property (3) $\Rightarrow L$ has property (3). - Now, assume that $L$ has property (3). Let $\left\{x_{n}: n=1,2, \cdots,\right\}$ be a sequence in $L_{\sigma(L, E)}$ such that $x_{n} \downarrow 0$. By Theorem 3.5, there exists a sequence $\left\{y_{n}: n=1,2, \cdots,\right\}$ in $L$ such that $y_{n} \downarrow 0$ and $x_{n} \leqq y_{n}$ for $n=1,2, \cdots$, . Since $L$ has property (3), there exists a sequence of real numbers $\quad\left\{0 \leqq \lambda_{n}: n=1,2, \cdots,\right\}$ with $\lambda_{n} \uparrow \infty$ such that $\lambda_{n} y_{n} \rightarrow 0$ (in $L$ ). But, $0 \leqq \lambda_{n} x_{n} \leqq \lambda_{n} y_{n}$ for all $n$. Hence, $\lambda_{n} x_{n} \rightarrow 0$ (in $\left.L_{\sigma(L, E)}\right)$. We have shown that if $L$ has property (3) then $L_{\sigma(L, E)}$ has property (3). The validity of (ii) is now established.

We will now establish that (iv) is valid. By [11, Th. 67.7(ii) p. 467], showing that a Riesz space $K$ has the Egoroff property is equivalent to showing that for every $u \in K^{+}$if $\left\{u_{n, k}: n, k=1,2, \cdots,\right\}$ is a double sequence in $K$ with $u \geqq u_{n, k} \downarrow_{k} 0$ then there exist integers $k(n)$ such that $u_{n, k(n)} \rightarrow 0$. Now suppose that $L_{\sigma(L, E)}$ has property (5). Let $u \in L^{+}$and let the double sequence $\left\{u_{n, k}: n, k=1,2, \cdots,\right\}$ in $L$ be such that $u \geqq$ $u_{n, k} \downarrow_{k} 0$ (in $L$ ). Then, $u \geqq u_{n, k} \downarrow_{k} 0$ (in $L_{\sigma(L . E)}$ ). Hence, there exist integers $k(n)$ such that $u_{n, k(n)} \rightarrow 0$ (in $L_{\sigma(L . E)}$ ). By Theorem 3.5, we have that $u_{n, k(n)} \rightarrow 0$ (in $L$ ). We have established that $L_{\sigma(L . E)}$ has property (5) $\Rightarrow L$ has property (5). Now, suppose that $L$ has property (5). Let $u \in L_{\sigma(L, E)}^{+}$, and let the double sequence $\left\{u_{n . k}: n, k=1,2, \cdots,\right\}$ in $L_{\sigma(L . E)}$ be such that $u \geqq u_{n, k} \downarrow_{k} 0$ for each $n$. Since $L_{\sigma(L . E)}$ is intermediate with respect to $L$, there exists a $u^{\prime} \in L$ such that $u^{\prime} \geqq u$. By virtue of Theorem 3.5, there exists, for each $n$, a sequence $\left\{u_{n, k}^{\prime}: k=1,2, \cdots,\right\}$ in $L$ with $u_{n, k}^{\prime} \downarrow_{k} 0$ (in $L$ ) such that $u_{n, k}^{\prime} \geqq u_{n, k}$ for $n, k=1,2, \cdots$, . Let the double sequence $\left\{u_{n . k}^{\prime \prime}: n, k=1,2, \cdots,\right\}$ in $L$ be defined by $u_{n . k}^{\prime \prime}=$ $u^{\prime} \wedge u_{n, k}^{\prime}$. Then, $u^{\prime} \geqq u_{n, k}^{\prime \prime} \downarrow_{k} 0 \quad$ (in $\left.L\right)$ and $u_{n, k} \leqq u_{n, k}^{\prime \prime}$ for $n, k=$ $1,2, \cdots$, . Since $L$ has property (5), there exist integers $k(n)$ such that $u_{n, k(n)}^{\prime \prime} \rightarrow 0$ (in $L$ ). Since $0 \leqq u_{n, k(n)} \leqq u_{n, k(n)}^{\prime \prime}$ for each $n$, we now have that $u_{n, k(n)} \rightarrow 0$ (in $\left.L_{\sigma(L, E)}\right)$. We have shown that $L$ has property $(5) \Rightarrow L_{\sigma(L . E)}$ has property (5). The validity of (iv) is established. To see that (iii) is valid, we first invoke [11, Th. 68.2 (ii), p. 470 and Th. 68.7 (i), p. 473] to see that showing a Riesz space $K$ has property (4) is equivalent to showing that if $\left\{u_{n, k}: n, k=1,2, \cdots,\right\}$ is a double sequence in $K$ such that, for each $n, u_{n, k} \downarrow_{k} 0$ then there exist integers $k(n)$ such that $L_{\sigma(L, L \bar{c})}=L_{o c}^{-}$remainder of the proof is almost exactly like the proof of (iv) except simplified by not having to bound the sequences $\left\{u_{n, k}^{\prime}\right\}$.

The theorem is proved.

Since, in general, $L_{\sigma\left(L, L_{c c}^{-}\right)}=L_{o c}^{-}$and $L_{\sigma\left(L, L \bar{r}_{u}\right)}=L_{r u}^{-}$, we have the following corollary as an immediate consequence of Theorem 6.3 and Lemma 6.1.

COROllary 6.4. Let $L$ be a Riesz space. Then $L$ has property $(r)$ if and only if $L_{o c}^{-}\left(L_{r u}^{-}\right)$has property $(r)$, where $r=1,2,3,4$ or 5. 
COROLlary 6.5. If $L$ is an order separable Riesz space and $E$ is any Riesz space intermediate with respect to $L$, then $L$ has property $(r)$ if and only if $E$ has property $(r)$, where $r=1,2,3,4$ or 5 .

Proof. By virtue of Corollary 3.13, $L_{\sigma(L, E)}=E$. The assertion now follows from Lemma 6.1 and Theorem 6.3.

In particular we have the following:

Corollary 6.6. If $L$ is an Archimedean, order separable Riesz space, then $L$ has property $(r)$ if and only if $L^{\wedge}$ has property $(r)$, where $r=1,2,3,4$ or 5 .

Corollary 6.6 above was proved by the author in [16, Prop. 1.1, p. 14 and Th. 1.3, p. 16]. The fact that if $L$ is order separable and Archimedean then $L$ has property (4) if and only if $L^{\wedge}$ has property (4) was proved in [4, Prop. 2.3 p. 160].

In $\$ 5$, we indicated that we did not know whether it was always true for Archimedean Riesz spaces that $L_{r u}=L_{(r u)^{2}}=L_{r u}^{-}$. This next result shows that if $L$ has certain of the properties we have been considering then this is the case.

Theorem 6.7. Let $L$ be an Archimedean Riesz space. If $L$ has property $(r)$, for $r=1,2,3$ or 4 , then $L_{r u}=L_{r u}^{-}$. Even more, if $L$ has property $(s)$, for $s=2,3$ or 4 , then $L_{r u}=L_{\sigma}$ and hence $L_{r u}^{-}=L_{o c}^{-}$.

Proof. We will first show that if $L$ has property (1) (the $\sigma$-property) then $L_{r u}=L_{r u}$. This follows in a straightforward manner by first applying Lemma 6.1 (i) to $L$ and $L^{\wedge}$, and then using [11, Th. 72.1 (iii), p. 487].

Since, when $L$ is Archimedean, $L_{\sigma}=L_{o c}^{-}$and $L_{o c}^{-}$is relatively uniform complete, the result will now follow if we can show that when $L$ has property $(s)$ for $s=2,3$ or 4 then $L_{r u}=L_{\sigma}$. But, by [11, Th. 16.3, p. 80 and Th. 70.2, p. 478], for Archimedean Riesz spaces property (2) is equivalent to property (3) and property (4) implies property (3). Hence, the result will follow if we can just show that $L$ has property (2) implies that $L_{r u}=L_{\sigma}$. Since $L_{r u} \subseteq L_{\sigma}$, we need only show that $L_{\sigma} \subseteq L_{r u}$. To this end, let $x \in \sigma(L)$. Then, there exist sequences $\left\{x_{n}^{\prime}\right\}$ and $\left\{x_{n}^{\prime \prime}\right\}$ in $L^{+}$such that $x_{n}^{\prime} \downarrow x$ (in $L_{\sigma}$ ) and $x_{n}^{\prime \prime} \uparrow x$ (in $L_{\sigma}$ ). But then $x_{n}^{\prime}-x_{n}^{\prime \prime} \downarrow 0$ (in $L_{\sigma}$ ). By Th. 6.3 (i), $L_{\sigma}=L_{\sigma\left(L, L^{\wedge}\right)}$ has property (2) if $L$ does. Hence, $x_{n}^{\prime}-x_{n}^{\prime \prime} \rightarrow 0(r \cdot u)$ (in $L_{\sigma}$ and, hence, in $L$ ). Thus, $x_{n}^{\prime} \rightarrow x(r \cdot u)$. We have shown that $L_{\sigma} \subseteq L_{r u}$. The theorem is proved.

REMARK 6.8. It is easy to see that if a Riesz space $L$ has a strong order unit (i.e., an element $0<x \in L$ such that if $y \in L$ there exists an $\alpha \in R$ such that $|y| \leqq \alpha x)$ then $L$ has the $\sigma$-property. 
EXAMPLE 6.9. If $L$ is taken to be the Archimedean Riesz space of Example 5.1, then $L$ is seen to have the $\sigma$-property, but $L_{r u}=L_{r u}^{-} \neq L_{\sigma}$.

We will now obtain two results which tend to indicate how far certain properties can be extended.

Theorem 6.10. Let $L$ be an Archimedean Riesz space and let $E$ be an intermediate space (with respect to $L$ ) such that $E \supseteq L_{u}$ (in particular, $E=L_{u}$ or $\left.L_{s}\right)$. Then, if $E$ has property $(r)$, for $r=2,3$ or 4 , $L_{\sigma}=L^{\prime}=L_{r u}$ and both $L$ and $L^{\prime}$ have property $(r)$.

Proof. By Lemma 6.1, Theorem 6.3 and Theorem 6.7, we will be done if we can show that if $E$ has property $(r)$, for $r=2,3$ or 4 , then $L_{\sigma}=L^{\prime}$. Again, since property (3) and property (2) are equivalent and property (4) implies property (3) (as cited in the proof of Theorem 6.7), we will be done if we show that $E$ having property (2) implies that $L_{\sigma}=L^{\prime}$. Suppose that $E$ has property (2). Let $x \in u(L)$ and let $\left\{x_{n}\right\}$ be a sequence in $L^{+}$such that $x_{n} \uparrow x$ (in $L^{\wedge}$ and, therefore, in $E$ ). Since $E$ has property (2) and is intermediate with respect to $L$, there exists a sequence $\left\{\lambda_{n}\right\} \subset R^{+}$with $\lambda_{n} \downarrow 0$ and a $y \in L^{+}$such that $\left|x-x_{n}\right| \leqq$ $\lambda_{n} y$. But then $x \in r u(L) \subseteq \sigma(L)$. Hence, $u(L) \subseteq \sigma(L) \subseteq l(L)$ and, by Theorem 4.5 (vi), this implies that $L_{\sigma}=L^{\prime}$. The theorem is proved.

REMARK 6.11. Let $L$ be a Riesz space. Let $x \in L_{s}^{+}$and let $\left\{x_{n}\right\}$ be a sequence in $L^{+}$such that $x_{n} \rightarrow x$ (in $L^{*}$ ). Then, $x_{n} \rightarrow x$ (in $L_{s}$ ). To see this consider, for each $n,\left\{x_{i}: i \geqq n\right\}=B_{n}$. Let $\left\{y_{n}\right\} \subset L^{*}$ be such that $y_{n} \downarrow 0$ and, for each $n,-y_{n} \leqq x_{n}-x \leqq y_{n}$. If $U$ is the set of all upper bounds in $L^{*}$ for $B_{n}$ then $\inf \{u-x: u \in U$ and $\left.x \in B_{n}\right\}=0$. This is true since, for each $i \geqq n, x+y_{i} \in U$ and $\inf \left\{x+y_{i}-x_{i}: i=n, n+1, \cdots\right\}=0$. But then $\sup B_{n}=s_{n}$ exists in $L^{*}$ and is in $L_{s}$. Similarly, one can show that, for each $n, \inf \left\{x_{i}: i \geqq n\right\}=$ $s_{n}^{\prime}$ exists in $L^{*}$ and is in $L_{s}$. Since $\left|x_{n}-x\right| \leqq s_{n}-s_{n}^{\prime} \downarrow 0$ we have that $x_{n} \rightarrow x$ (in $L_{s}$ ).

Theorem 6.12. Let $L$ be a Riesz space and let $E$ be intermediate with respect to $L$. Furthermore, assume that $E \supseteq L_{s u}=\left(L_{s}\right)_{u}$. Then, if $E$ has the Egoroff property (property (5)), $L_{s}=L^{\prime}$.

Proof. We will show that $u\left(L_{s}\right) \subseteq L_{s}$ which clearly implies the result. To this end, let $x \in u\left(L_{s}\right)$ and let $\left\{x_{n}\right\}$ be a sequence in $L_{s}$ such that $x_{n} \uparrow x$ (in $L^{*}$ and, hence, in $E$ ). For each $n$, let $\left\{x_{n, m}: m=1,2, \cdots,\right\}$ be a sequence in $L^{+}$such that $x_{n, m} \rightarrow{ }_{m} x_{n}$ (in $L^{*}$ ). By virtue of Remark 6.11, we have that $x_{n, m} \rightarrow_{m} x_{n}$ (in $L_{s}$ and, hence, in $E$ ). Let $y \in L$ be such that $y \geqq x$. For each $n, m$, let $x_{n, m}^{\prime}=x_{n, m} \wedge y$. Then, for each $n, m, x_{n, m}^{\prime} \in L^{+}, 0 \leqq x_{n, m}^{\prime} \leqq y$ and $x_{n, m}^{\prime} \rightarrow_{m} x_{n}^{\prime}$ (in $E$ ). By [11, Th. 69.1 
(iii), p. 476], $E$ has the Egoroff property implies that there exist integers $m(n)$ such that $x_{n, m(n)}^{\prime} \rightarrow x$ (in $E$ and thus in $L^{*}$ ). This says that $x \in L_{s}$. The theorem is proved.

Since the Egroff property is implied by the diagonal property, the following result is a direct consequence of Theorem 6.12 .

Corollary 6.13. Let $L$ be a Riesz space and let $E$ be a Riesz space which is intermediate with respect to $L$. Furthermore, assume that $E \supseteq L_{s u}$. Then, if $E$ has the diagonal property, $L_{s}=L^{\prime}$.

7. Some examples. In this section, we give two examples which better illustrate the limitations of Lemma 6.1 in the preceding section.

This first example shows, in particular, that, even if $L$ is assumed to be Archimedean, the implications in parts (ii), (iii) and (iv) of Lemma 6.1 cannot be reversed.

ExAmPLE 7.1. Let $L$ be the Archimedean Riesz space of Example 5.2. It was shown in [4, Ex. 1 pp. 161-162] that $L$ has the diagonal property (and, hence, that $L$ has property $(r)$ for $r=2,3$ or 4 ). By means of Theorem 6.7, we see that $L_{r u}=L_{\sigma}$. We showed, in Example 5.2 , that $L_{\sigma} \neq L^{\prime}$. Thus, it follows from Theorem 6.10 that no intermediate Riesz space containing $L_{u}=L^{\prime}$ (in this case) has property $(r)$ for $r=2,3$ or 4 .

This next example shows that the Archimedean assumption in parts (iii) and (iv) of Lemma 6.1 cannot be dropped.

EXAMPLE 7.2. For each countable ordinal $1 \leqq \alpha<\Omega(\Omega=$ first uncountable ordinal), let $E_{\alpha}$ be the lexicographically ordered plane. Let $E^{\prime}=\prod_{1 \leqq \alpha<\Omega} E_{\alpha} \quad$ (with the usual coordinate wise ordering). Let $E_{b}^{\prime}$ be the set of all $x=\left(x_{\alpha}\right)=\left(\begin{array}{l}x_{\alpha}^{1} \\ x_{\alpha}^{2}\end{array}\right)$ in $E^{\prime}$ such that there exists a real number $m>0$ such that, for all $1 \leqq \alpha<\Omega$, we have $\left|x_{\alpha}^{1}\right| \leqq m$ and $\left|x_{\alpha}^{2}\right| \leqq m$. Let $L$ be the Riesz subspace of $E_{b}^{\prime}$ consisting of those elements $\left(\begin{array}{l}x_{\alpha}^{1} \\ x_{\alpha}^{2}\end{array}\right)=\left(x_{\alpha}\right)$ such that $\left(x_{\alpha}\right)$ is at most countably non-constant and $\left(x_{\alpha}^{1}\right)$ is at most finitely nonconstant. Finally, let $E$ be the Riesz subspace of $E_{b}^{\prime}$ consisting of those elements $\left(x_{\alpha}\right)=\left(\begin{array}{l}x_{\alpha}^{1} \\ x_{\alpha}^{2}\end{array}\right)$ such that;

(a) $\left(x_{\alpha}\right)$ is at most countably nonconstant, and

(b) there exists a real number $r$ such that for every $\epsilon>0$ $\left\{\alpha:\left|x_{\alpha}^{1}-r\right| \geqq \epsilon\right\}$ is finite.

We will show first that $E_{b}^{\prime}$ is intermediate with respect to $L$. From this it will follow that $E$ is also intermediate with respect to $L$. So, let 
$\left(x_{\alpha}\right)=\left(\begin{array}{l}x_{\alpha}^{1} \\ x_{\alpha}^{2}\end{array}\right)$ be in $E_{b}^{\prime}$. Let the real number $m \geqq 0$ be such that $\left|x_{\alpha}^{1}\right| \leqq m$ and $\left|x_{\alpha}^{2}\right| \leqq m$ for all $\alpha$. For each $\alpha$, define $\left(u_{\beta}^{\alpha}\right)$ and $\left(v_{\beta}^{\alpha}\right)$ in $L$ by:

$$
\begin{aligned}
& u_{\beta}^{\alpha}=\left(\begin{array}{l}
x_{\alpha}^{1} \\
x_{\alpha}^{2}
\end{array}\right) \text { for } \beta=\alpha \text { and } u_{\beta}^{\alpha}=\left(\begin{array}{c}
m \\
m
\end{array}\right) \text { for } \beta \neq \alpha, \\
& v_{\beta}^{\alpha}=\left(\begin{array}{l}
x_{\alpha}^{1} \\
x_{\alpha}^{2}
\end{array}\right) \text { for } \beta=\alpha \text { and } v_{\beta}^{\alpha}=\left(\begin{array}{c}
-m \\
-m
\end{array}\right) \text { for } \beta \neq \alpha .
\end{aligned}
$$

Clearly, $\inf \left\{\left(u_{\beta}^{\alpha}\right): 1 \leqq \alpha<\Omega\right\}=\sup \left\{\left(v_{\beta}^{\alpha}\right): 1 \leqq \alpha<\Omega\right\}=\left(x_{\alpha}\right)$. This is enough to establish that $E_{b}^{\prime}$ (and, thus, $E$ ) is intermediate with respect to $L$.

Next, we wish to show that $E$ has the diagonal property. Before we can accomplish this, we must first make a number of observations concerning order convergence in $E$.

(1) If $y=\left(y_{\alpha}\right) \in E$, there exists an $\alpha(y)<\Omega$ such that if $\alpha \geqq \alpha(y)$ then $y_{\alpha}^{1}=y_{\alpha(y)}^{1}$ and $y_{\alpha}^{2}=y_{\alpha(y)}^{2}$. This is a trivial consequence of the fact that $\left(y_{\alpha}\right)$ is at most countably nonconstant.

(2) If $\left\{y_{n}: n=1,2, \cdots\right\}$ is a sequence in $E^{+}$such that $y_{n} \downarrow 0$, then, for each $\alpha$, there exists an $n(\alpha)$ such that, for all $n \geqq n(\alpha), y_{n, \alpha}^{1}=0$. To see this, suppose to the contrary that there exists an $\alpha$ such that, for all $n, y_{n, \alpha}^{1}>0$. Let $x=\left(x_{\beta}\right) \in E$ be defined by,

$$
\begin{aligned}
& x_{\beta}=\left(\begin{array}{l}
0 \\
1
\end{array}\right) ; \text { for } \beta=\alpha, \\
& x_{\beta}=\left(\begin{array}{l}
0 \\
0
\end{array}\right) ; \text { for } \beta \neq \alpha .
\end{aligned}
$$

Then, $0<x \leqq y_{n}$ for all $n$. Hence, $y_{n} \nrightarrow 0$.

(3) If $\left\{y_{n}: n=1,2, \cdots,\right\}$ is a sequence in $E^{+}$such that $y_{n} \downarrow 0$, then there exist an integer $N$ and a $\beta<\Omega$ such that, if $n \geqq N$ and $\alpha \geqq \beta$, then $y_{n, \alpha}^{1}=0$. To see this, let $\alpha\left(y_{n}\right)$ be the ordinal associated with $y_{n}$ by (1) above. Suppose that, for all $n, y_{n, \alpha\left(y_{n}\right)}^{1}>0$. Let $\beta<\Omega$ be such that $\beta>\alpha\left(y_{n}\right)$ for $n=1,2, \cdots$, . Then, $y_{n, \beta}^{1}>0$ for all $n$. This cannot happen by virtue of (2) above.

(4) If $\left\{y_{n}: n=1,2, \cdots\right\}$ is a sequence in $E^{+}$such that $y_{n} \downarrow 0$, then there exist a $\beta<\Omega$ and a sequence of real numbers $\left\{\lambda_{n}: n=1,2, \cdots,\right\}$ with $\lambda_{n} \downarrow 0$ such that $y_{n, \alpha}^{2} \leqq \lambda_{n}$ for $n=1,2, \cdots$, and for all $\alpha \geqq \beta$. The proof of this is straightforward (from (1)) and is omitted.

We are now ready to show that $E$ has the diagonal property. As was cited in the proof of part (iv) of Theorem 6.3, it suffices to show that if $\left\{y_{n, m}: n, m=1,2, \cdots,\right\}$ is a double sequence in $E$ such that, for each $n, y_{n, m} \downarrow_{m} 0$ then there exists a sequence of integers $m(n)$ such that 
$y_{n, m(n)} \rightarrow 0$. So, let $\left\{y_{n, m}: n, m=1,2, \cdots,\right\}$ be a double sequence in $E$ such that, for each $n, y_{n, m} \downarrow_{m} 0$. For each $n$, let $\beta(n)<\Omega$ and let $m_{1}(n)$ be such that, for all $\alpha \geqq \beta(n)$ and $m \geqq m_{1}(n)$, we have $y_{n, m, \alpha}^{\prime}=0$ (by (3) above we can do this). Let $\beta^{\prime}(n)$ and the real sequences $\left\{\lambda_{n, m}: n, m=\right.$ $1,2, \cdots$,$\} be such that, for \alpha \geqq \beta^{\prime}(n), y_{n, m, \alpha}^{2} \leqq \lambda_{n, m} \downarrow_{m} 0$ (that we can do such follows from (4) above). Let, now, $\beta<\Omega$ be chosen so that $\beta>\beta(n)$ and $\beta \geqq \beta^{\prime}(n)$ for all $n=1,2, \cdots$, . Then, for all $\alpha \geqq \beta$, we have

(i) $y_{n, m, \alpha}^{1}=0$ for all $n$ and $m \geqq m_{1}(n)$, and

(ii) $y_{n, m, \alpha}^{2} \leqq \lambda_{n, m}$ for all $n, m$.

For each $n=1,2, \cdots$, there exists an $m_{2}(n)$ such that, for $m \geqq m_{2}(n)$, $\left\{\alpha: y_{n, m, \alpha}^{1} \geqq \frac{1}{n}\right\}$ is finite. It follows then from (2) above that, for each $n$, there exists an $m_{3}(n)$ such tht $y_{n, m, \alpha}^{1}<\frac{1}{n}$ for all $m \geqq m_{3}(n)$. Now, let $f:\{1,2, \cdots,\} \rightarrow\{1 \leqq \alpha<\beta\}$ be a one-to-one correspondence, and let $\alpha_{n}$ denote $f(n)$. For each $n$, let $m_{4}(n)$ be chosen so that, if $m \geqq m_{4}(n)$, $y_{n, m, \alpha_{t}}^{1}=0$ for $i=1,2, \cdots, n$. Furthermore, for each $n$, let $m_{5}(n)$ be chosen so that, if $m \geqq m_{5}(n), y_{n, m, \alpha_{i}}^{2} \leqq \frac{1}{n}$, for $i=1,2, \cdots, n$. Finally, for each $n=1,2, \cdots$, let $m(n)$ be chosen so that $m(n) \geqq \max \left\{m_{j}(n): j=\right.$ $1,2,3,4,5\}$ and $\lambda_{n, m(n)} \leqq \frac{1}{n}$. We claim that $y_{n, m(n)} \rightarrow 0$. To see this, let the sequence $\left\{x_{n}\right\}$ in $E$ be defined by

$$
\begin{array}{ll}
x_{n, \alpha}=\left(\begin{array}{c}
\frac{1}{n+k} \\
0
\end{array}\right) ; & \text { for } \alpha=\alpha_{n+k,} k=1,2, \cdots, \\
x_{n, \alpha}=\left(\begin{array}{c}
0 \\
\frac{1}{n}
\end{array}\right) ; & \text { otherwise. }
\end{array}
$$

Clearly, $x_{n} \downarrow 0$ and, for each $n, y_{n, m(n)} \leqq x_{n}$. We have established that $E$ has the diagonal property.

We will now show that order convergence is not stable in $L$. Let the sequence $\left\{y_{n}: n=1,2, \cdots,\right\}$ in $L$ be defined by

$$
\begin{aligned}
& y_{n, \alpha}=\left(\begin{array}{l}
0 \\
1
\end{array}\right) ; \text { for } n<\alpha<\omega, \\
& y_{n, \alpha}=\left(\begin{array}{l}
0 \\
0
\end{array}\right) ; \text { otherwise. }
\end{aligned}
$$

We note that $y_{n} \downarrow 0$ in $L$. Let $\left\{\lambda_{n}: n=1,2, \cdots\right\}$ be any sequence of positive real numbers such that $\lambda_{n} \uparrow \infty$. We will show that 
$\lambda_{n} y_{n} \not \supset 0$. To see this, suppose first that $y \in L$ is an upper bound for $\left\{\lambda_{n} y_{n}: n \geqq N\right\}$ where $N$ is some positive integer. Then, there must exist an integer $m(y) \geqq 1$ such that, if $m(y) \leqq \alpha<\omega$, then $y_{\alpha}^{1}=y_{m(y)}^{1}>$ 0 . But then there must exist also a $\beta(y)<\Omega$ such that, for all $\alpha \geqq \beta(y)$, we have $y_{\alpha}^{1}=y_{m(y)}^{1}>0$. Thus, if $\left\{z_{n}: n=1,2, \cdots\right\}$ is any sequence in $L$ with $z_{n} \downarrow$ such that, for each $n, \lambda_{n} y_{n} \leqq z_{n}$; we must have, for each $n$, a $\beta(n)<\Omega$ such that, if $\alpha \geqq \beta(n)$, then $z_{n, \alpha}^{1}=z_{n, \beta(n)}^{1}>0$. If $\beta<\Omega$ is chosen so that $\beta \geqq \beta(n)$ for $n=1,2, \cdots$, then, for all $\alpha \geqq \beta$ and for all $n$, we have that $z_{n, \alpha}^{1}=z_{n, \beta}^{1}>0$. But then by (2) above $\inf \left\{z_{n}: n=1,2, \cdots,\right\} \neq 0$. This says that $\lambda_{n} y_{n} \nrightarrow 0$. We have shown that order convergence is not stable in $L$.

Next, we will show that $L$ does not have the Egoroff property. Let $\left\{y_{n}: n=1,2, \cdots,\right\}$ be the sequence defined in (*). Consider, for each $n=1,2, \cdots$, the sequence $\left\{n y_{k}: k=1,2, \cdots,\right\}$ in $L$. Clearly, $n y_{k} \downarrow_{k} 0$, for each $n$. Let $y \in L$ be defined by $y_{\alpha}=\left(\begin{array}{l}1 \\ 0\end{array}\right)$, for all $\alpha$. Obviously, for each $n, k=1,2, \cdots$, we have that $n y_{k} \leqq y$. If we could find for each $n$ a $k(n)$ such that $n y_{k(n)} \rightarrow 0$ in $L$, then taking $\lambda_{i}=n$, for each $k(n) \leqq i<k(n+1)$, we would have that $\lambda_{i} y_{i} \rightarrow 0$ and $\lambda_{i} \uparrow \infty$ (which we have already shown is impossible). Hence, $L$ does not have the Egoroff property.

It is not difficult to show, by a proof similar to that used above, that if a Riesz space has the diagonal property then order convergence is stable in it. It is immediate from [11, Th. 67.7 (ii), p. 467] that the diagonal property implies the Egoroff property. Thus, we have shown the following:

(A) Order convergence is stable in $E$ but not in $L$.

(B) $E$ has the diagonal property, but $L$ does not have the diagonal property.

(C) $E$ has the Egoroff property, but $L$ does not have the Egoroff property.

Question: If $E$ and $L$ are Archimedean Riesz spaces with $E$ intermediate with respect to $L$, is it true that if $E$ has the Egoroff property then $L$ has the Egoroff property?

This next example shows that the Archimedean assumption in Theorem 6.10 cannot be released.

ExAmple 7.3. Let $L$ and $E$ be the Riesz spaces of Example 7.2 above. Let $L_{1}$ be the Riesz subspace of $L$ consisting of those $\left(x_{\alpha}\right)=\left(\begin{array}{l}x_{\alpha}^{1} \\ x_{\alpha}^{2}\end{array}\right)$ such that both $\left(x_{\alpha}^{1}\right)$ and $\left(x_{\alpha}^{2}\right)$ are finitely non-constant. It can be shown that $L_{1 \sigma}$ is the set of all $\left(x_{\alpha}\right)$ in $L$ for which there exists a real number $r=r\left(\left(x_{\alpha}\right)\right)$ such that, for every real number $\epsilon>0$, 
$\left\{\alpha:\left|r-x_{\alpha}^{2}\right| \geqq \epsilon\right\}$ is finite. It can also be seen that $L_{1}^{\prime}=L_{s}=L$. Thus $E \supseteq L_{s}$ and $E$ has the diagonal property but $L_{1 \sigma} \neq L_{1}^{\prime}$.

8. Almost complete and almost $\sigma$-complete spaces. We will say the Riesz space $L$ is almost complete (almost $\sigma$-complete) if and only if $L_{o c}^{-}=L_{\sigma}=L^{*}\left(L_{o c}^{-}=L_{\sigma}=L^{\prime}\right)$.

In this section we begin by giving an intrinsic characterization of the almost $\sigma$-complete Riesz spaces. We will then give sufficient conditions involving certain projection properties for an Archimedean Riesz space to be almost complete or almost $\sigma$-complete. We do not know of a necessary and sufficient condition that a Riesz space be almost complete.

Remark 8.1. Every order separable Riesz space is almost complete (see Theorem 4.1).

Definition 8.2. The Riesz space $L$ is said to be strongly order cauchy (order cauchy) if whenever $\left\{x_{n}\right\}$ is a decreasing sequence in $L^{+}$ (such that $\inf \left\{x_{n}-v: n=1,2, \cdots, v \in V\right\}=0$ where $V$ is the set of lower bounds in $L$ of $\left.\left\{x_{n}\right\}\right)\left\{x_{n}\right\}$ is order cauchy.

Using Proposition 2.7 and Theorem 4.4 (v) it is not difficult to establish the following result.

THEOREM 8.3. The Riesz space L is almost $\sigma$-complete if and only if $L$ is order cauchy.

REMARK 8.4. It is not difficult to see that a strongly order cauchy Riesz space must be Archimedean. Indeed, if $L$ is a strongly order cauchy Riesz space and $y \in L^{+}$, then $\left\{\frac{1}{n} y\right\}$ is an order cauchy sequence. Hence, $\frac{1}{n(n+1)} y=\left(\frac{1}{n} y-\frac{1}{n+1} y\right) \downarrow 0$. This is enough to establish that $\frac{1}{n} y \downarrow 0$. We have the following corollary.

Corollary 8.5. The Riesz space L is Archimedean and almost $\sigma$-complete if and only if $L$ is strongly order cauchy.

In what follows, a knowledge of Chapters 3 and 5 of [11] is assumed. For the convenience of the reader, we will state a few definitions here.

An ideal $I$ in a Riesz space $L$ is said to be a principal ideal if there exists $e \in I^{+}$such that for every $x \in I$ there exists an $\alpha \in R$ such that $|x| \leqq \alpha e$. 
A band (order closed ideal) in a Riesz space $L$ is said to be a projection band if it is a direct summand of $L$.

$A$ band $B$ in $L$ is said to be the principal band generated by. $0<e \in L$ if $B$ is the smallest band containing the principal ideal generated by $e$ (in this case we denote $B$ by $B_{e}$ ).

A Riesz space $L$ is said to have the projection property (P.P.) if every band in $L$ is a projection band.

A Riesz space $L$ is said to have the principal projection property (P.P.P.) if every principal band in $L$ is a projection band.

We will denote the boolean algebra of projection bands (the lattice of principal projection bands) by $P(L)\left(P_{p}(L)\right)$.

We say that $P_{p}(L)$ is Dedekind- $\sigma$-complete if whenever $\left\{B_{u_{1}}: i=\right.$ $1,2, \cdots$,$\} is a sequence in P_{p}(L)$ with $B_{u_{t}} \uparrow$ and such that there exists $B_{u} \in P_{p}(L)$ with $B_{u_{i}} \subseteq B_{u}$, for all $i$, then there exists $B_{r} \in P_{p}(L)$ such that $B_{u} \uparrow B_{r}$.

If $B_{u} \in P_{p}(L)$ and $v \in L$, then we will denote the projection onto $B_{u}$ of $v$ by $P_{u}(v)$.

Let $a<b$ be real numbers. By $\Pi=\Pi\left(\alpha_{0}, \cdots, \alpha_{n}\right)$ where $a=\alpha_{0}<$ $\alpha_{1}<\cdots<\alpha_{n}=b$, we denote a partition of the closed interval $[a, b]$.

Let $a<b$ be real numbers, let $L$ be a Riesz space with the P.P.P., and let $e, f \in L$ be such that, for some $\epsilon>0$, $a e \leqq f \leqq(b-\epsilon) e$. For any partition $\Pi\left(\alpha_{0}, \alpha_{1}, \cdots, \alpha_{n}\right)$ of $[a, b]$ we define $s(\Pi ; f)$ and $t(\Pi ; f)$ by

$$
s(\Pi ; f)=\sum_{k=1}^{n} \alpha_{k-1}\left(P_{(\alpha k e-f)}+(e)-P_{(\alpha k-1 e-f)}+(e)\right)
$$

and

$$
t(\Pi ; f)=\sum_{k=1}^{n} \alpha_{k}\left(P_{(\alpha k e-f)^{+}}(e)-P_{(\alpha k-1 e-f)^{+}}(e)\right)
$$

We have the following result:

THEOREM 8.6. If the Riesz space L has the P.P.P. and the property that $P_{p}(L)$ is Dedekind- $\sigma$-complete, then $L$ is Archimedean and $L_{r u}=L^{\prime}$.

Proof. Let $L$ be a Riesz space with the P.P.P. and the property that $P_{p}(L)$ is Dedekind- $\sigma$-complete. Since $L$ has the P.P.P., $L$ is Archimedean (c.f., [11, Th. 30.4, p. 174]). Let $x \in l(L)$ and let $\left\{u_{n}: n=0,1,2, \cdots,\right\}$ be a sequence in $L$ such that $u_{n} \downarrow x$ (in $L^{\wedge}$ ). We will show that, for any real number $-\infty<\alpha<\infty, \quad P_{\left(\alpha u_{0}-x\right)^{+}}\left(u_{0}\right)=$ $\sup \left\{m\left(\alpha u_{0}-x\right)^{+} \wedge u_{0}: m=1,2, \cdots\right\}\left(\right.$ taken in $\left.L^{\wedge}\right)$ is an element of $L$. To see this, consider, for each $n=1,2, \cdots,\left(\alpha u_{0}-u_{n}\right)^{+} \in L$. We have, for $n=1,2, \cdots, \quad$ that $\left(\alpha u_{0}-u_{n}\right)^{+} \leqq\left(\alpha u_{0}-u_{n+1}\right)^{+}$. Also, $\left(\alpha u_{0}-u_{n}\right)^{+}$ $\uparrow\left(\alpha u_{0}-x\right)^{+}\left(\right.$in $\left.L^{\wedge}\right)$. Thus, by virtue of [11, Cor. 31.2, p. 182], we have 
the $P_{\left(\alpha u_{0}-u_{n}\right)^{+}}\left(u_{0}\right) \uparrow P_{\left(\alpha u_{0}-x\right)^{+}}\left(u_{0}\right)$ (in $\left.L^{\wedge}\right)$. Now, for each $n=1,2, \cdots$, the principal projection hand $B_{\left(\alpha u_{0}-u_{n}\right)^{+}} \subseteq B_{u_{0}}$ (this in $\left.P_{p}(L)\right)$, and $B_{\left(\alpha u_{0}-u_{n}\right)^{+}} \uparrow$. Since $L$ has the property that $P_{p}(L)$ is Dedekind- $\sigma$ complete, there exists a $v \in L^{+}$such that $B_{\left(\alpha u_{0}-u_{n}\right)}+\uparrow B_{r}$ (in $\left.P_{p}(L)\right)$. By [11, Cor. 31.2, p. 182], we have that

$$
P_{\left(\alpha u_{0}-u_{n}\right)^{+}}\left(u_{0}\right) \uparrow P_{v}\left(u_{0}\right)
$$

(in $L$ and, hence in $\left.L^{\wedge}\right)$. But then, $P_{\left(\alpha u_{0}-x\right)^{+}}\left(u_{0}\right)=$ $P_{\mathrm{r}}\left(u_{0}\right) \in L$, which is the desired result. Now, since $u_{0} \geqq x \geqq 0$ we have that $(3 / 2-1 / 2) u_{0} \geqq x \geqq 0\left(u_{0}\right)$. Let $\Pi_{n}$, for $n=1,2, \cdots$, be a sequence of partitions of the interval $[0,3 / 2]$ such that $\Pi_{n+1}$ is a refinement of $\Pi_{n}$, for each $n$, and $\left|\Pi_{n}\right| \leqq \frac{1}{n}$ (where, by $\left|\Pi_{n}\right|$ we mean the length of the largest subinterval between consecutively indexed points of the partition $\left.\Pi_{n}\right)$. Let $s\left(\Pi_{n} ; x\right)$ and $t\left(\Pi_{n} ; x\right)$ be defined as above. It follows from what we have already shown that, for each $n, s\left(\Pi_{n} ; x\right) \in L$ and $t\left(\Pi_{n} ; x\right) \in L$. Furthermore, it follows from the Freudenthal spectral theorem (see $\left[11\right.$, th. 40.2, p. 257]) that $\left|t\left(\Pi_{n} ; x\right)-x\right| \leqq \frac{1}{n} u_{0}$ and $\left|s\left(\Pi_{n} ; x\right)-x\right| \leqq \frac{1}{n} u_{0}$, for all $n$ (this theorem is being applied in $\left.L^{\wedge}\right)$. This says that $x \in r u(L)$. We have shown that $l(L) \subseteq r u(L) \subseteq$ $u(L)$. It now follows from Theorem 4.4 that $L_{r u}=L_{\sigma}=L^{\prime}$. The theorem is proved.

Corollary 8.7. If the Riesz space $L$ has the P.P.P. and the property that $P_{p}(L)$ is Dedekind- $\sigma$-complete, then $L$ is Archimedean and almost $\sigma$-complete.

This next result is the analogue of Theorem 8.6 for the case that $L$ has the projection property.

THEOREM 8.8. If the Riesz space $L$ has the P.P., then $L$ is Archimedean and $L_{r u}=L^{\wedge}$.

Proof. Since $L$ has the P.P., it has the P.P.P. and is Archimedean. Let $0<\hat{x} \in L^{\wedge}$ and let $x \in L$ be such that $x \geqq \hat{x}$. It follows from [8, Th. 2.6 (iii), p. 309] that, for every real number $-\infty<\alpha<\infty, P_{(\alpha x-x)^{+}}(x)$ (taken in $L^{\wedge}$ ) is in $L$. The remainder of the proof now follows exactly as it did in the proof of Theorem 8.6. The theorem is proved.

Corollary 8.9. If the Riesz space $L$ has the P.P., then $L$ is Archimedean and almost complete. 
Example 8.10. Let $L=C([0,1])$ (the Archimedean Riesz space of continuous real valued functions defined on $[0,1])$. It is known that $L$ is order separable (c.f., [11, Exercise 29.6, p. 170]) but fails to have even the P.P.P. . Thus, $L$ is an example of an almost complete Riesz space without the P.P.P.

Question. If $L_{r u}=L^{\wedge}\left(L_{r u}=L^{\prime}\right)$ does $L$ have to have the P.P. (the P.P.P. and the property that $P_{p}(L)$ is Dedekind- $\sigma$-complete)?

REMARK 8.11. A. I. Veksler introduced in [17] the concept of an $r$-completion for Archimedean Riesz spaces which agrees with our $L_{r u}$ when $L$ is Archimedean. Furthermore, he obtained results which directly imply Theorem 8.8 and Corollary 8.9 above.

REMARK 8.12. Independently, the authors of [2] have defined a concept which, for Archimedean Riesz spaces, is equivalent to the concept of almost $\sigma$-complete Riesz spaces introduced in this section.

9. Some topological considerations. A certain theorem due to Nakano has been of interest to a number of researchers (c.f., [9, Note $\mathrm{XVI}_{\mathrm{B}}$ ] and [15, Prop. 2.6, p. 164]). Nakano's theorem states that if $(L,\|\cdot\|)$ is a Dedekind- $\sigma$-complete normed vector lattice with the property that a sequence $\left\{y_{n}: n=1,2, \cdots,\right\}$ in $L$ norm converges to zero whenever $y_{n} \downarrow 0$, then $L$ is order separable. The result in [15] mentioned above is a generalization of Nakano's theorem to the setting of topological vector lattices. There are examples of integrally normed Riesz spaces (i.e., a normed vector lattice $L_{\rho}$ with norm $\rho$ such that $\rho\left(u_{n}\right) \rightarrow 0$ whenever $u_{n} \downarrow 0$ ) which are not order separable (c.f., [4, Ex. 1, p. 161]).

If $E$ is a vector lattice, a subset $W$ of $E$ is said to be solid if $x \in W$ whenever $y \in W$ and $|x| \leqq|y|$. If $E$ is a topological vector space with a neighborhood base at zero consisting of solid sets, then $E$ is said to be a topological vector lattice. The notation $(E, \tau)$ will refer to a topological vector space with topology $\tau$.

This next theorem can be viewed as a completion to the result in [15].

The reader should keep in mind that, since metrizable topological vector lattices are Archimedean, the concepts of order cauchy and strongly order cauchy are equivalent in this setting.

THEOREM 9.1. Let $(E, \tau)$ be a metrizable topological vector lattice with the property that $u_{n} \stackrel{\tau}{\rightarrow} 0$ whenever $u_{n} \downarrow 0$. Then, E is order separable if and only if $E$ is strongly order cauchy. 
Proof. It follows from Remark 8.1 and Corollary 8.5 that if $E$ is order separable then $E$ is strongly order cauchy. We wish now to show that if $E$ is strongly order cauchy then $E$ is order separable. To this end, let $\left\{W_{n}: n=1,2, \cdots,\right\}$ be a countable neighborhood base at zero for $\tau$ consisting of full, circled sets $W_{n}$ such that $W_{n+1}+W_{n+1} \subset W_{n}$ for all $n$. Let $\left\{x_{\alpha}\right\}$ be a net in $E$ such that $x_{\alpha} \downarrow 0$. We wish to show that there exists a sequence $\left\{x_{\alpha_{k}}\right\} \subseteq\left\{x_{\alpha}\right\}$ such that $x_{\alpha_{t}} \downarrow 0$. We note first that, for each $n=1,2, \cdots$, there exists an $\alpha$ such that for all $\beta \geqq \alpha$ we have $x_{\alpha}-x_{\beta} \in W_{n}$. Suppose not, then there exists an $n_{0}$ and a sequence $x_{\alpha_{m}}$ such that $x_{\alpha_{m}}-x_{\alpha_{m+1}} \notin W_{n_{0}}$. But then $x_{\alpha_{m}}-x_{\alpha_{m+1}} \nrightarrow 0$ (since $x_{\alpha_{m}}-x_{\alpha_{m+1}} \stackrel{\tau}{\neq} 0$ ). Since $E$ is strongly order cauchy, this is a contradiction. Let the sequence $\left\{x_{\alpha_{n}}\right\} \subseteq\left\{x_{\alpha}\right\}$ be chosen so that $x_{\alpha_{n}} \downarrow$ and, for all $\beta \geqq \alpha_{n}, x_{\alpha_{n}}-x_{\beta} \in W_{n}$. Suppose that $\inf _{n}\left\{x_{\alpha_{n}}\right\} \neq 0$. Then there exists a $0<v \leqq x_{\alpha_{n}}$ for all $n=1,2, \cdots$, . For each $m=1,2, \cdots$, and each $\beta \geqq \alpha_{m}$, we have $x_{\alpha_{m}}-\left(x_{\beta} \wedge v\right)=\left(x_{\alpha_{m}}-x_{\beta}\right) \vee\left(x_{\alpha_{m}}-v\right) \leqq$ $\left(x_{\alpha_{m}}-x_{\beta}\right)+\left(x_{\alpha_{m}}-v\right)$. Hence,

$$
x_{\alpha_{m}}-x_{\beta} \geqq v-\left(x_{\beta} \wedge v\right) .
$$

Since $v>0$ and $x_{\beta} \downarrow 0$, there exists a $\beta^{\prime}$ such that, for all $\beta \geqq \beta^{\prime}$

$$
v-\left(x_{\beta} \wedge v\right) \geqq v-\left(x_{\beta^{\prime}} \wedge v\right)>0 .
$$

But then there exists an $n^{\prime}$ such that for all $\beta \geqq \beta^{\prime}$ we have that $v-x_{\beta} \wedge v \notin W_{n}^{\prime}$. Let $\beta_{1} \geqq \beta^{\prime}$ be such that $\beta_{1} \geqq \alpha_{n}^{\prime}$. Then, $x_{\alpha_{n}}-x_{\beta_{1}} \notin W_{n^{\prime}}$ which contradicts the choice of $\alpha_{n}^{\prime}$. Hence, we must have that $\inf \left\{x_{\alpha_{n}}: n=1,2, \cdots,\right\}=0$. The theorem is proved.

REMARK. A topological vector lattice $(E, \tau)$ is said to have property $(A, i)$ if, whenever $u_{n} \downarrow 0$ in $L$, it follows that $u_{n} \stackrel{\tau}{\rightarrow} 0$. It is said to have property $(A, i i)$ if for any net $u_{\alpha} \downarrow 0$ it follows that $u_{\alpha} \stackrel{\tau}{\rightarrow} 0$. It is said to have property $(A, i i i)$ if, whenever $0 \leqq u_{n} \uparrow \leqq u$, it follows that $u_{n}$ is a $\tau$-Cauchy sequence. In [1], $\mathrm{C}$. $\mathrm{D}$. Aliprantis has shown that a metrizable topological vector lattice having properties $(A, i)$ and $(A$, iii $)$ must have property $(A, i i)$. It is easy to see that a topological vector lattic with property $(A, i i)$ is order separable. Thus another proof of Theorem 9.1 can be obtained by noting that $(A, i)$ and strongly order cauchy imply $(A, i)$ and $(A, i i i)$ and then using the above comments.

It has been of interest to discover which combinations of topological and order properties imply the diagonal property (c.f., [4, Prop. 3.3, p. 164] and [15, Prop. 2.6, p. 164]). 
The result in [15] involved some completeness properties which were relaxed in [4]. Unfortunately, the result in [4] required some topological assumptions which were more stringent than those of the result in [15]. In both cases, order separability was either implied by the assumptions or actually assumed.

We will say that the topological vector lattice $(E, \tau)$ is $\sigma$-monotone bounded if every topologically bounded monotone increasing sequence in $E$ is order bounded.

Compare this next result with the above cited results in [4] and [15].

THEOREM 9.2. Let $(E, \tau)$ be a metrizable, $\sigma$-monotone bounded topological vector lattice with the property that $x_{n} \stackrel{\tau}{\rightarrow} 0$ whenever $x_{n} \downarrow 0$. Then, E has the diagonal property.

Proof. We will show first that the conditions of the theorem imply that $E$ has the $\sigma$-property (i.e., if $\left\{x_{n}\right\} \subset E^{+}$there exist a sequence $\left\{\lambda_{n}\right\}$ of positive real numbers and an $x \in E$ such that, for each $n, x_{n} \leqq$ $\left.\lambda_{n} x\right)$. To this end, let $\left\{x_{n}\right\}$ be a sequence in $E^{+}$. Let $\left\{W_{n}: n=1,2, \cdots,\right\}$ be a neighborhood base at zero for $\tau$ consisting of solid sets such that $W_{n+1}+W_{n+1} \subset W_{n}$ for each $n$. Consider, for each $n$, the sequences $\frac{1}{k} x_{n}$ where $k=1,2, \cdots$, Since $\frac{1}{k} x_{1} \stackrel{\tau}{\rightarrow} 0$, there exists a $k(1)$ such that $\frac{1}{k(1)} x_{1} \in W_{1}$. Since $W_{1}$ is open and $\frac{1}{k} x_{2} \stackrel{\tau}{\rightarrow} 0$, there exists a $k(2)$ such that $\frac{1}{k(2)} x_{2} \in W_{2}$ and $\sup \left\{\frac{1}{k(1)} x_{1}, \frac{1}{k(2)} x_{2}\right\} \in W_{1}$. By induction, for each $n$, there exists a $k(n)$ such that $\frac{1}{k(n)} x_{n} \in W_{n}$ and $1 \leqq j \leqq n-1$ then

$$
\sup \left\{\frac{1}{k(i)} x_{i}: j \leqq i \leqq n\right\} \in W_{j}
$$

Let, for each $n, z_{n}$ be defined by

$$
z_{n}=\sup \left\{\frac{1}{k(i)} x_{i}: 1 \leqq i \leqq n\right\}
$$

Then, $z_{n} \uparrow$. We wish to show that $\left\{z_{n}\right\}$ is topologically bounded. To this end, let $m$ be a positive integer and consider $W_{m}$. Then,

$$
z_{n}=z_{m} \vee\left(\sup \left\{\frac{1}{k(i)} x_{i}: m+1 \leqq i \leqq n\right\}\right)
$$

for $n \geqq m+1$. Now, 


$$
\sup \left\{\frac{1}{k(i)} x_{i}: m+1 \leqq i \leqq n\right\} \in W_{m+1}
$$

for all $n \geqq m+1$ and $z_{m} \in E$. Hence, there exists a $t \geqq 1$ such that $z_{m} \in t W_{m+1}$. But then $z_{n} \in t W_{m}$ for all $n$. This establishes that $\left\{z_{n}\right\}$ is topologically bounded. Since $(E, \tau)$ is $\sigma$-monotone bounded, there exists a $y \in E$ such that $z_{n} \leqq y$ for all $n$. Thus, for each $n, x_{n} \leqq$ $k(n) y$. We have established that $e$ has the $\sigma$-property.

Now suppose that $x_{n} \downarrow 0$. By hypothesis, this implies that $x_{n} \stackrel{\tau}{\rightarrow} 0$. Hence, there exists an $n(1)$ such that $x_{n(1)} \in W_{1}$. Since $W_{1}$ is open and $2 x_{n} \stackrel{r}{\rightarrow} 0$, there exists an $n(2)>n(1)$ such that $2 x_{n(2)} \in W_{2}$ and $\sup \left\{x_{n(1)}, 2 x_{n(2)}\right\} \in W_{1}$. By induction, there exists, for each integer $k$, an integer $n(k)>n(k-1)$ such that $x_{n(k)} \in W_{k}$ and, for each $1 \leqq j \leqq k-1$,

$$
\sup \left\{i x_{n(i)}: j \leqq i \leqq k\right\} \in W_{j}
$$

Letting, for each $k, z_{k}=\sup \left\{i x_{n(i)}: 1 \leqq i \leqq k\right\}$ we have that $z_{k} \uparrow$ and that $\left\{z_{k}\right\}$ is topologically bounded. Hence, there exists a $y \in E$ such that $y \geqq z_{k} \geqq k x_{n(k)}$ for each $k=1,2, \cdots$, . Thus, $x_{n(k)} \leqq \frac{1}{k} y$ for $k=$ $1,2, \cdots$, . For $n(k) \leqq n<n(k+1)-1$, let $\lambda_{n}=\frac{1}{k}$. Then, $\lambda_{n} \downarrow 0$ and $x_{n} \leqq \lambda_{n} y$ for $n=1,2, \cdots$, . We have shown that $x_{n} \rightarrow 0(r \cdot u)$ whenever $x_{n} \downarrow 0$. Since the hypothesis $((E, \tau)$ is a metrizable topological vector lattice) implies that $E$ is Archimedean, this is enough to show that relatively uniform convergence and order convergence for sequences are equivalent in $E$.

We have seen that $E$ is Archimedean, has the $\sigma$-property, and has the property that relatively uniform convergence and order convergence for sequences are equivalent. By virtue of $[11$, Th. 16.3, p. 80 and Th. 70.2, p. 478], this implies that $E$ has the diagonal property. The theorem is proved.

10. Some structure space considerations. In [6] several characterizations of the projection property in Archimedean Riesz spaces were given. The approach primarily explored the interrelationships between such things as the band structure or the prime ideal structure of an Archimedean Riesz space and corresponding structures of its Dedekind completion. These structures were compared by means of the following natural method (first introduced in [10]) of associating ideals in the Dedekind completion of an Archimedean Riesz space with ideals in the Riesz space. 
If $L$ is an Archimedean Riesz space and $L^{\wedge}$ is its Dedekind completion then to each ideal $I$ in $L$ is corresponded the ideal $I^{\wedge}$ in $L^{\wedge}$ where $I^{\wedge}=\left\{\hat{x} \in L^{\wedge}\right.$ : there exists $y \in I$ with $\left.|\hat{x}| \leqq y\right\}$.

It should be clear that this correspondence, suitably modfied, will make perfectly good sense in the setting of intermediate Riesz spaces. It is the purpose of this section to study the relationship between prime ideal structure in a Riesz space $L$ and prime ideal structure in a Riesz space $E$ which is intermediate with respect to $L$. The results we obtain in this section not only generalize some of the results in [8] but they also tend to clarify the relationships between several of the properties underlying the results in [8].

We begin our investigation with some definitions and a notational agreement. For the theory of prime ideals the reader is referred to [6] or [11].

An ideal $P$ in the Riesz space $L$ is prime if $x \wedge y$ in $P$ implies $x$ is in $P$ or $y$ is in $P$. Equivalently, $P$ is a prime ideal in $L$ if $x \wedge y=0$ implies either $x$ is in $P$ or $y$ is in $P$.

A subset $K$ of the positive cone of a Riesz space $L$ is called a lower sublattice if $x \wedge y$ is in $K$ whenever $x$ and $y$ are in $K$.

A prime ideal $P$ is called a minimal prime ideal if $P$ does not properly contain any other prime ideal.

It was shown in [6] that, if one partially orders by inclusion the lower sublattices in a Riesz space which do not intersect a given ideal, then every lower sublattice which does not intersect this ideal is contained in a maximal lower sublattice which does not intersect the ideal. Furthermore, it was shown that the ideal $P$ contained in the Riesz space $L$ is a minimal prime ideal if and only if $L^{+}-P$ is a maximal lower sublattice not intersecting $\{0\}$.

If $L$ and $E$ are Riesz spaces with $E$ intermediate with respect to $L$ and if $I$ is an ideal in $L$ then by $I(E)$ we will denote the ideal in $E$ defined by $I(E)=\{e \in E$ : there exists $y \in I$ with $|e| \leqq y\}$.

Definition 10.1. Let $L$ and $E$ be Riesz spaces with $E$ intermediate with respect to $L$. It will be said that $L$ has the prime extension property with respect to $E$ if $P(E)$ is prime in $E$ whenever $P$ is a prime ideal in $L$.

THEOREM 10.2. Let $L$ and $E$ be Riesz spaces with E intermediate with repsect to $L$. Then the following conditions are equivalent:

(i) L has the prime extension property with respect to $E$.

(ii) If $P$ is a minimal prime ideal in $L$ then $P(E)$ is a minimal prime ideal in $E$.

(iii) If $P$ is a minimal prime ideal in $L$ then $P(E)$ is a prime ideal in E. 
(iv) If $e_{1}, e_{2} \in E^{+}$are such that $e_{1} \wedge e_{2}=0$, there exist $y_{1}, y_{2} \in L$ with $y_{1} \geqq e_{1}$ and $y_{2} \geqq e_{2}$ such that $y_{1} \wedge y_{2}=0$.

Proof. We begin by showing that (i) $\Rightarrow$ (ii). Let $P$ be a minimal prime ideal in $L$. By virtue of condition (i) we have that $P(E)$ is a prime ideal in $E$. If $M$ is a prime ideal in $E$ such that $M \subseteq P(E)$ then $M \cap L$ is prime in $L$ and $M \cap L \subseteq P=P(E) \cap L$. Since $P$ is a minimal prime ideal in $L$, it follows that $M \cap L=P$. But, then $P(E)=(M \cap L)(E) \subseteq M$. Hence, $M=P(E)$ and $P(E)$ is seen to be a minimal prime ideal in $E$. We have established that (i) $\Rightarrow$ (ii).

That (ii) $\Rightarrow$ (iii) is obvious. We show now that (iii) $\Rightarrow$ (iv). Suppose that condition (iv) is not satisfied. Let $e_{1}, e_{2} \in E$ be such that $e_{1} \wedge e_{2}=0$ and such that $y_{1} \wedge y_{2}>0$ whenever $y_{1}, y_{2} \in L, y_{1} \geqq e_{1}$ and $y_{2} \geqq e_{2}$. Let $K \subset L^{+}$be defined by

$$
K=\left\{z \in L^{+}: z=y_{1} \wedge y_{2} \text { for some } y_{1}, y_{2} \in L \text { with } y_{1} \geqq e_{1} \text { and } y_{2} \geqq e_{2}\right\} \text {. }
$$

By construction, $0 \notin K$. We claim that $K$ is a lower sublattice. To see this, let, for $i=1,2, z_{i}=y_{\imath} \wedge x_{i}$ where $\left\{x_{i}, y_{i}: i=1,2\right\} \subset L, y_{i} \geqq e_{1}$ and $x_{i} \geqq e_{2}$. Then, $z_{1} \wedge z_{2}=\left(y_{1} \wedge y_{2}\right) \wedge\left(x_{1} \wedge x_{2}\right)$ where $y_{1} \wedge y_{2} \geqq e_{1}$ and $x_{1} \wedge x_{2} \geqq$ $e_{2}$. Thus, $z_{1} \wedge z_{2} \in K$ and this establishes that $K$ is a lower sublattice in $L$. Now, let $K^{\prime}$ be a lower sublattice of $L$ which is maximal with respect to containing $K$ and not containing $\{0\}$. Let $P=P^{+}-P^{+}$where $P^{+}=L^{+}-K^{\prime}$. Then $P$ is a minimal prime ideal in $L$. We note now that if $e_{1} \leqq y \in L$ then $y \in K$ and, hence, $y \notin P$. Indeed, if $e_{1} \leqq y \in L$ and $e_{2} \leqq y_{2} \in L$ then $y=y \wedge\left(y \vee y_{2}\right)$. Similarly, if $e_{2} \leqq x \in L$ then $x \notin P$. But, then $e_{1} \notin P(E)$ and $e_{2} \notin P(E)$. Since $e_{1} \wedge e_{2}=0$, this establishes that $P(E)$ is not prime. We have established that (iii) $\Rightarrow$ (iv).

All that remains to be shown is that (iv) $\Rightarrow$ (i). To this end, let $P$ be a prime ideal in $E$ and consider $P(E)$. Let $e_{1}, e_{2} \in E^{+}$be such that $e_{1} \wedge e_{2}=0$. By virtue of condition (iv) there exist $y_{1}, y_{2} \in L$ with $y_{1} \geqq e_{1}$ and $y_{2} \geqq e_{2}$ such that $y_{1} \wedge y_{2}=0$. Since $P$ is prime in $L$, we must have that $y_{1} \in P$ or $y_{2} \in P$. This implies that $e_{1} \in P(E)$ or $e_{2} \in P(E)$ and this implies that $P(E)$ is prime.

The theorem is proved.

Compare conditions (i) and (iii) of this next lemma with conditions (iii) and (iv) of Theorem 2.6 in [8].

Lemma 10.3. Let $L$ and $E$ be Riesz spaces with $E$ intermediate with respect to $L$. Consider the following conditions:

(i) For any $e \in E^{+}$there exists an $x \in L$ with $x \geqq e$ such that $\{x\}^{\perp \perp}-$ in $-E=\{e\}^{\perp \perp}-$ in $-E \quad$ (where $\quad\{x\}^{\perp}-$ in $-E=$ $\{y \in E:|y| \wedge|x|=0\}$ and $\{x\}^{\perp \perp}-$ in $-E=\left\{\{x\}^{\perp}-\text { in }-E\right\}^{\perp}-$ in $\left.-E\right)$. 
(ii) For any $e_{1}, e_{2} \in E^{+}$with $e_{1} \wedge e_{2}=0$ there exist $y_{1}, y_{2} \in L$ with $y_{1} \wedge y_{2}=0$ such that $y_{1} \geqq e_{1}$ and $y_{2} \geqq e_{2}$.

(iii) Given any $x \in L^{+}$and $\bar{x} \in E$ with $\bar{x} \wedge(x-\bar{x})=0$, it follows that $\bar{x} \in L$.

Then, (i) $\Rightarrow$ (ii) $\Rightarrow$ (iii). Furthermore, if $E$ has the P.P.P. (principal projection property), then (iii) $\Rightarrow$ (i) and all the condition are equivalent.

Proof. Establishing that (i) $\Rightarrow$ (ii) is routine. Showing that if $E$ has the P.P.P. then (iii) $\Rightarrow$ (i) is also not difficult and we omit the proof. We are left with showing that (ii) $\Rightarrow$ (iii). To this end, let $x \in L^{+}$and let $\bar{x} \in E$ be such that $\bar{x} \wedge(x-\bar{x})=0$. From condition (ii) it follows that there exist $y_{1}, y_{2} \in L$ with $y_{1} \geqq \bar{x}$ and $y_{2} \geqq(x-\bar{x})$ such that $y_{1} \wedge y_{2}=0$. Consider $y_{1} \wedge x$. We have immediately that $y_{1} \wedge x \geqq$ $x \wedge \bar{x}=\bar{x}$. Since $y_{1} \wedge y_{2}=0$ and $y_{2} \geqq(x-\bar{x})$, we have that $y_{1} \wedge(x-\bar{x})=$ 0 and, therefore, that $\left(y_{1} \wedge x\right) \wedge(x-\bar{x})=0$. Since $y_{1} \wedge x \leqq x$ and $(x-\bar{x}) \leqq x$, we have the $\left(y_{1} \wedge x\right)+(x-\bar{x})=\left(y_{1} \wedge x\right) \vee(x-\bar{x}) \leqq x=$ $\bar{x}+(x-\bar{x})$. Therefore, $y_{1} \wedge x \leqq \bar{x}$. Hence, $\bar{x}=y_{1} \wedge x \in L$. We have established that (ii) $\Rightarrow$ (iii). The lemma is proved.

Lemma 10.4. Let $L$ be an Archimedean Riesz space. Then the following are equivalent :

(i) $L$ has the P.P.P. and the property that $P_{p}(L)$ is Dedekind- $\sigma$ complete.

(ii) If $x \in L^{+}$and $\bar{x} \in L^{\prime}$ are such that $\bar{x} \wedge(x-\bar{x})=0$, then $\bar{x} \in L$.

Proof. We will show first that (i) $\Rightarrow$ (ii). Let $x \in L^{+}$and suppose that $\bar{x} \in L^{\prime}$ is such that $\bar{x} \wedge(x-\bar{x})=0$. By virtue of Theorem 8.6, we have that there exists a sequence $\left\{u_{n}\right\} \subset L^{+}$such that $u_{n} \uparrow \bar{x}$. Consider now the sequence of principal projection bands $\left\{B_{u_{n}}\right\} \subset P_{p}(L)$. We have that $B_{u_{n}} \uparrow$ and $B_{u_{n}} \subseteq B_{x}$ for $n=1,2, \cdots$, . Since $P_{p}(L)$ is Dedekind- $\sigma$-complete, there exists $v \in L^{+}$such that $B_{u_{n}} \uparrow B_{r}$. Since $u_{n} \uparrow \bar{x}$, we have that $P_{u_{n}}(x) \uparrow P_{\bar{x}}(x)=\bar{x}$ (where these projections are taken in $L^{\prime}$ ) (see [11, Cor. 31.2, p. 183]). Since $L$ has the P.P.P., $P_{u_{n}}(x) \in L$ for $n=1,2, \cdots$, . Since $B_{u_{n}} \uparrow B_{r}$, we have $P_{u_{n}}(x) \uparrow P_{r}(x) \in$ $L$ (see [11, Cor. 31.2, p. 183]. Hence, $\bar{x}=P_{r}(x) \in L$. We have established that (i) $\Rightarrow$ (ii).

We will now show that (ii) $\Rightarrow$ (i). To this end, let $u, x \in L^{+}$. Since $L^{\prime}$ is Dedekind- $\sigma$-complete, it follows that $\sup _{n}\{n u \wedge x: n=1,2, \cdots\}$ exists in $L^{\prime}$. Let

$$
\bar{x}=\sup \{n u \wedge x: n=1,2, \cdots,\},
$$

where this supremum is taken in $L^{\prime}$. It follows from [11, Th. 24.7 (i), p. 135] that $\bar{x} \wedge(x-\bar{x})=0$. Hence, $\bar{x} \in L$. Thus, by virtue of [11, Th. 
24.9(iii), p. 137], we have established that $L$ has the P.P.P.. Now, let $\left\{B_{u_{n}}\right\}_{n=1}^{\infty}$ be a sequence in $P_{p}(L)$ such that $B_{u_{n}} \uparrow$ and there exists $v \in L^{+}$ such that $B_{u_{n}} \subseteq B_{v}$ for all $n$. For each $n$, let $v_{n}=P_{u_{n}}(v)$. Then, $\left\{v_{n}\right\} \subset L^{+}, v_{n} \uparrow$ and $v_{n} \leqq v$ for $n=1,2, \cdots$, . Since $L^{\prime}$ is Dedekind- $\sigma$ complete, there exists a $\bar{v}$ such that $\omega_{n} \uparrow \bar{v}$. By [11, Cor. 31.2, p. 182] we have that $P_{v_{n}}(v)=v_{n} \uparrow P_{\bar{v}}(v)$ (where these projections are taken in $\left.L^{\prime}\right)$. But $v_{n} \uparrow \bar{v}$ and thus $P_{\bar{v}}(v)=\bar{v}$. Hence, $\bar{v} \wedge(v-\bar{v})=0$ which implies (by (ii)) that $\bar{v} \in L$. Applying [11, Cor. 31.2, p. 182] again, we see that $B_{u_{n}}=B_{v_{n}} \uparrow B_{\bar{v}}$ in $P_{p}(L)$. We have shown that $P_{p}(L)$ is Dedekind- $\sigma$-complete.

The assertion is proved.

The proof of this next result provides an alternate method of proving one of the principle results in [8].

Theorem 10.5. Let $L$ be an Archimedean Riesz space. Then $L$ has the prime extension property with respect to $L^{\wedge}\left(L^{\prime}\right)$ if and only if $L$ has the projection property ( $L$ has the principal projection property and the property that $P_{p}(L)$ is Dedekind-o-complete).

Proof. The assertion that $L$ has the prime extension property with respect to $L^{\wedge}$ if and only if $L$ has the P.P. follows from Theorem 10.2, Lemma 10.3 and [8, Lemma 2.3, p. 308]. The assertion that $L$ has the prime extension property with respect to $L^{\prime}$ if and only if $L$ has the P.P.P. and the property that $P_{p}(L)$ is Dedekind- $\sigma$-complete follows from Theorem 10.2, Lemma 10.3 and Lemma 10.4. The theorem is proved.

Theorem 10.6. Let $L$ be an Archimedean Riesz space. Then, $L$ has the prime extension property with respect to $L_{u}$ if and only if $L$ has the P.P.P. and the property that $P_{p}(L)$ is Dedekind-o-complete.

Proof. The sufficiency follows from Theorem 8.6 and Theorem 10.5 We are left with proving the necessity. So, assume that $L$ has the prime extension property with respect to $L_{u}$. Let $0<x \in l(L)$ and let $x<u \in L$. Let $\alpha$ be any real number and consider $v_{\alpha}=$ $(\alpha u-x)^{+}$. Since $x \in l(L)$ and $u \in L, v_{\alpha}$ is in $u(L)$.

It is straightforward now to show that $P_{v_{\alpha}}(u)=$ $\sup \left\{n v_{\alpha} \wedge u: n=1,2, \cdots,\right\}$ is in $u(L)$ (the projection being taken in $\left.L^{\wedge}\right)$. Since $P_{v_{\alpha}}(u) \wedge\left(u-P_{v_{\alpha}}(u)\right)=0$, it follows now, by virtue of Theorem 10.2 and Lemma 10.3 , that $P_{v_{\alpha}}(u) \in L$. It now follows in exactly the same way as in the proof of Theorem 8.6 that $x \in$ $r u(L)$. We have shown that $l(L) \subseteq r u(L) \subseteq u(L)$. This is enough to imply that $L_{u}=L_{r u}=L^{\prime}$. But, then, $L$ has the prime extension property with respect to $L^{\prime}$. Thus, by Theorem $10.5, L$ has the P.P.P. and the property that $P_{p}(L)$ is Dedekind- $\sigma$-complete. The theorem is proved. 
We give now an example which shows that the conditions in Lemma 10.3 need not be equivalent if the assumption that $e$ has the P.P.P. is dropped.

EXAmPLE 10.5. Let $L$ be the Archimedean Riesz space of finitely nonconstant real sequences. Let $E=c$ be the Riesz space of convergent real sequences. Clearly $E$ is intermediate with respect to $L$. Let $e_{1}=(1,0,1 / 3,0,1 / 5, \cdots)$ and let $e_{2}=$ $(0,1 / 2,0,1 / 4,0,1 / 6, \cdots)$. Then $e_{1}, e_{2} \in E^{+}$and $e_{1} \wedge e_{2}=0$. Suppose that $\left(y_{i}\right)=y \in L$ is such that $y \geqq e_{1}$. Then there must exist an integer $n(y)$ such that, for all integers $m \geqq n(y), y_{m}=y_{n(y)}>0$. Similarly, if $\left(x_{i}\right)=x \in L$ is such that $x \geqq e_{2}$ then there exists an $n(x)$ such that, for $j \geqq n(x), x_{j}=x_{n(x)}>0$. This is enough to show that if $e_{1} \leqq y \in L$ and $e_{2} \leqq x \in L$ then $x \wedge y>0$. Thus, for this $L$ and $E$, condition (ii) (and, hence, condition (i)) of Lemma 10.3 is not satisfied.' It is not difficult to show, however, that condition (iii) of Lemma 10.3 is satisfied.

We do not know whether or not conditions (i) and (ii) of Lemma 10.3 are equivalent.

REMARK 10.6. The equivalence of the first three conditions in Theorem 10.2 is established in Lemma 3.2 of [10]. The fact that if $L$ has the prime extension property with respect to $L^{\wedge}$ then $L$ has the P.P. (Theorem 10.5) is implied by Theorem 3.9 of [10]. The proof given there is quite different either from that given here or from that in [8]. Theorem 3.9 in [10] also implies that if $E$ and $L$ are Archimedean and $E$ has the P.P.P. then so does $L$.

REMARK 10.7. Suppose that in this section we had not demanded that $E$ be intermediate with respect to $L$ but rather had only required that $L$ be a Riesz subspace of $E$ such that, for every $e \in E$, there exists a $y \in L$ such that $|e| \leqq y$. It is then implied by Theorem 3.9 of [10] that if the Archimedean Riesz space $L$ has the prime extension property with respect to such an $E$ and if $E$ has the P.P.P. then $E$ is intermediate with respect to $L$. The reader who is interested in a development of the prime extension property in the setting of distributive lattices should refer himself to [10].

\section{REFERENCES}

1. C. D. Aliprantis, On order and topological properties of Riesz spaces, Thesis, California Institute of Technology, 1973.

2. C. D. Aliprantis and Eric Langford, Almost $\sigma$-Dedekind complete Riesz spaces and the main inclusion theorem, to appear, Proc. Amer. Math. Soc.

3. B. Banashewski, Über die Vervollständigung geordneter Gruppen, Math. Nachr., 16 (1957), 51-71. 
4. G. Crofts and J. J. Masterson, Vector lattices and sequences spaces, Illinois J. Math., 16 (1972), 158-165.

5. C. J. Everett, Sequence completion of lattice moduls, Duke Math. J., 11 (1944), 109-119.

6. D. G. Johnson and J. E. Kist, Prime ideals in vector lattices, Canad. J. Math., 14 (1962), 517-528.

7. K. Kuratowski, Topology, Vol. I, Academic Press, New York, N.Y. (1968).

8. K. K. Kutty and J. Quinn, Some characterizations of the projection property in Archimedean Riesz spaces, Canad. J. Math., 24 (1972), 306-311.

9. W. A. J. Luxemburg, Notes on Banach function spaces, Proc. Acad. Sci. Amsterdam: Note $\mathrm{XVI}_{\mathrm{A}}, \mathrm{A68}, 646-657$ (1965); Note $\mathrm{XVI}_{\mathrm{B}}, \mathrm{A68}, 658-666$.

10 . Extensions of prime ideals and the existence of projections in Riesz spaces, Proc. Acad. Sci. Amsterdam, A76, 263-279 (1973).

11. W. A. J. Luxemburg and A. C. Zaanen, Riesz Spaces, I, North Holland-American Elsevier, Amsterdam-New York (1971).

12. H. M. MacNeille, Partially ordered sets, Trans. Amer. Math. Soc., 42 (1937), 416-460.

13. J. J. Masterson, Structures spaces of a vector lattice and its Dedekind completion, Proc. Acad. Sci. Amsterdam, A71, (1968), 468-478.

14. F. Papangelou, Order convergence and topological completion of commutative lattice-groups, Math. Ann., 155 (1964), 81-107.

15. A. L. Peressini, Ordered Topological Vector Spaces, Harper \& Row, New York, Evanston, and London (1967).

16. J. Quinn, Measure Riesz spaces and the Egoroff theorem, Thesis, Michigan State University (1970).

17. A. I. Veksler, Concerning a new construction of the Dedekind completion of a vector lattice and quotient spaces of l-groups, Sibiriskii Mat. Z., 10 (1969), 1206-1213, (in Russian).

Received July 22, 1973 and in revised form April 18, 1974. This research was partially supported by a UNCC Faculty Research Grant.

The University of North Carolina at Charlotte 




\section{Pacific Journal of Mathematics}

\section{Vol. 56, No. $1 \quad$ November, 1975}

Shimshon A. Amitsur, Central embeddings in semi-simple rings .......... 1

David Marion Arnold and Charles Estep Murley, Abelian groups, A, such

that $\operatorname{Hom}(A,---)$ preserves direct sums of copies of $A \ldots \ldots \ldots .$.

Martin Bartelt, An integral representation for strictly continuous linear

operators ................................... 21

Richard G. Burton, Fractional elements in multiplicative lattices......... 35

James Alan Cochran, Growth estimates for the singular values of

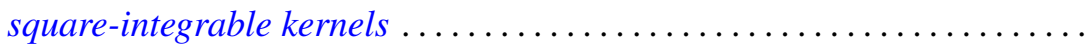

C. Martin Edwards and Peter John Stacey, On group algebras of central

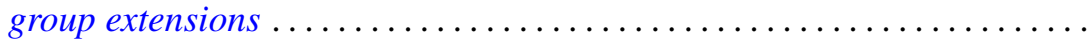

Peter Fletcher and Pei Liu, Topologies compatible with homeomorphism

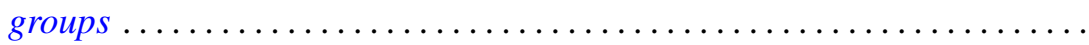

George Gasper, Jr., Products of terminating ${ }_{3} F_{2}(1)$ series ............ 87

Leon Gerber, The orthocentric simplex as an extreme simplex ............

Burrell Washington Helton, A product integral solution of a Riccati

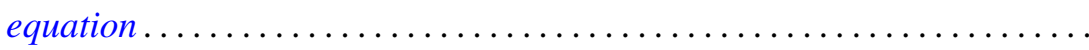

Melvyn W. Jeter, On the extremal elements of the convex cone of

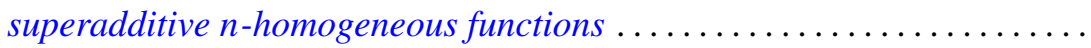

R. H. Johnson, Simple separable graphs .

Margaret Humm Kleinfeld, More on a generalization of commutative and

alternative rings. . .

A. Y. W. Lau, The boundary of a semilattice on an $n$-cell.

Robert F. Lax, The local rigidity of the moduli scheme for curves ...

Glenn Richard Luecke, A note on quasidiagonal and quasitriangular

operators .

Paul Milnes, On the extension of continuous and almost periodic functions

Hidegoro Nakano and Kazumi Nakano, Connector theory.

James Michael Osterburg, Completely outer Galois theory of perfect rings ..................................

Lavon Barry Page, Compact Hankel operators and the F. and M. Riesz

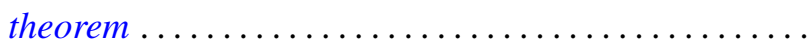

Joseph E. Quinn, Intermediate Riesz spaces..................... 225

Shlomo Vinner, Model-completeness in a first order language with a generalized quantifier.

Jorge Viola-Prioli, On absolutely torsion-free rings ..........

Philip William Walker, A note on differential equations with all solutions of integrable-square............................

Stephen Jeffrey Willson, Equivariant maps between representation 